Supporting Information for:

\title{
A Strategy for C-H Activation of Pyridines: Direct C-2 Selective Alkenylation of Pyridines by Nickel/Lewis-acid Catalysis
}

\author{
Yoshiaki Nakao,* Kyalo Stephen Kanyiva, and Tamejiro Hiyama* \\ Department of Material Chemistry, Graduate School of Engineering, \\ Kyoto University, Kyoto 615-8510 Japan
}

General. All manipulations of oxygen- and moisture-sensitive materials were conducted with a standard Schlenk technique or in a dry box under an argon atmosphere. Flash column chromatography was performed using Kanto Chemical silica gel (spherical, 40-50 $\mu \mathrm{m}$ ). Analytical thin layer chromatography (TLC) was performed on Merck Kieselgel $60 \mathrm{~F}_{254}(0.25 \mathrm{~mm})$ plates. Visualization was accomplished with UV light $(254 \mathrm{~nm})$ and/or an aqueous alkaline $\mathrm{KMnO}_{4}$ solution followed by heating.

Apparatus. Proton and carbon nuclear magnetic resonance spectra $\left({ }^{1} \mathrm{H}\right.$ NMR and ${ }^{13} \mathrm{C}$ NMR) were recorded on a Varian Mercury $400\left({ }^{1} \mathrm{H}\right.$ NMR, $400 \mathrm{MHz} ;{ }^{13} \mathrm{C}$ NMR, $\left.101 \mathrm{MHz}\right)$ spectrometer with solvent resonance as the internal standard ( ${ }^{1} \mathrm{H} \mathrm{NMR}, \mathrm{CHCl}_{3}$ at $7.26 \mathrm{ppm} ;{ }^{13} \mathrm{C} \mathrm{NMR}, \mathrm{CDCl}_{3}$ at 77.0 ppm). ${ }^{1} \mathrm{H}$ NMR data are reported as follows: chemical shift, multiplicity $(\mathrm{s}=$ singlet, $\mathrm{d}=$ doublet, $\mathrm{t}=$ triplet, $\mathrm{q}=$ quartet, quint $=$ quintet, sext $=$ sextet, $\mathrm{br}=$ broad, $\mathrm{m}=$ multiplet $)$, coupling constants $(\mathrm{Hz})$, and integration. Fluorine nuclear magnetic resonance spectra $\left({ }^{19} \mathrm{~F}\right.$ NMR) were recorded on a Varian Mercury $300(282 \mathrm{MHz})$ spectrometer with $\mathrm{CFCl}_{3}(0 \mathrm{ppm})$ as the internal standard. Melting points were determined using a YANAKO MP-500D. Elemental analyses were performed by Elemental Analysis Center of Kyoto University. High-resolution mass spectra were obtained with a JEOL JMS-700 (EI) or JEOL JMS-HX110A (FAB+) spectrometer. Preparative recycling gel permeation chromatography (GPC) and preparative recycling silica gel chromatography were performed with a JAI LC-908 chromatograph equipped with JAIGEL-1H and $-2 \mathrm{H}$ (chloroform as an eluent) and JAIGEL-SIL or Nacalai tesque 5SL-II (hexane-ethyl acetate as an eluent). GC analysis was performed on a Shimadzu GC 2014 equipped with an ENV-1 column (Kanto Chemical, $30 \mathrm{~m}$ x $0.25 \mathrm{~mm}$, pressure $=31.7 \mathrm{kPa}$, detector $=\mathrm{FID}, 290^{\circ} \mathrm{C}$ ) with helium gas as a carrier.

Chemicals. Unless otherwise noted, commercially available pyridines and alkynes were used after distillation. Anhydrous toluene was purchased from Kanto Chemical and degassed by purging vigorously with argon for $20 \mathrm{~min}$ and further purified by passage through activated alumina under positive argon pressure as described by Grubbs et al. ${ }^{1}$ 4-(Tert-butyldimethylsilyloxy)methylpyridine (1d) was prepared according to the literature procedure. ${ }^{2}$

Nickel-Lewis acid catalyzed hydroheteroarylation of alkynes. A general procedure. To a solution of pyridine (3.0 mmol), a Lewis acid [ $60 \mu \mathrm{mol}, \mathrm{ZnMe}_{2}: 60 \mu \mathrm{L}$ (a $1.00 \mathrm{M}$ solution in hexane); $\mathrm{ZnPh}_{2}$ (92\% purity from Aldrich): $14.3 \mathrm{mg} ; \mathrm{ZnPh}_{2}$ (100\% purity from Strem): $13.2 \mathrm{mg} ; \mathrm{AlMe}_{3}: 53 \mu \mathrm{L}$ (a $1.03 \mathrm{M}$ solution in hexane)], and an alkyne $(1.0 \mathrm{mmol})$ in toluene $(1.25 \mathrm{~mL})$ was added a solution of $\mathrm{Ni}(\mathrm{cod})_{2}(8.2 \mathrm{mg}, 30 \mu \mathrm{mol})$ and $\mathrm{P}(i-\mathrm{Pr})_{3}(19.2 \mathrm{mg}, 0.12 \mathrm{mmol})$ in toluene $(1.25 \mathrm{~mL})$ in a dry box. After addition of undecane (internal standard, $78 \mathrm{mg}, 0.50 \mathrm{mmol}$ ), the vial was taken outside the dry box and heated at the temperature for the time both specified in Tables 1 and 2. The resulting mixture was filtered through a silica gel pad, concentrated in vacuo, and purified by flush silica gel chromatography to give the corresponding products in yields listed in Tables 1 and 2. In some cases, $\mathbf{3}$ and $\mathbf{4}$ were separated by preparative recycling GPC or silica gel chromatography. Full details for the screening of 
Lewis acid catalysts are shown in Table S1. The spectra of 3aa and 3ia agreed well with those reported by us recently. ${ }^{3}$

Table S1. Direct C-2 Alkenylation of 1a with 2a Catalyzed by Nickel-Lewis acid ${ }^{a}$

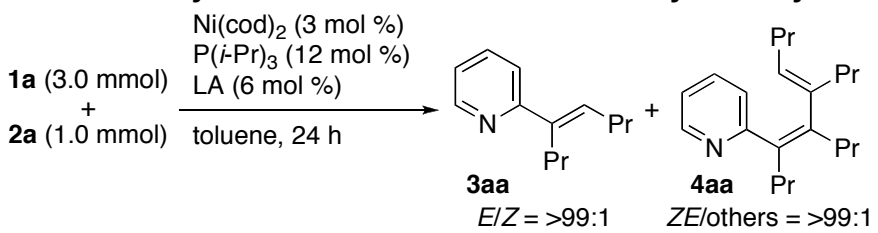

\begin{tabular}{clllc}
\hline entry & Lewis acid (LA) & temp $\left({ }^{\circ} \mathrm{C}\right)$ & yield of 3aa $(\%)^{a}$ & yield of 4aa $(\%)^{a}$ \\
\hline 1 & none & 50 & $<1$ & $<1$ \\
$2^{b}$ & $\mathrm{ZnMe}_{2}$ & 50 & $<1$ & $<1$ \\
$3^{b, c}$ & $\mathrm{ZnMe}_{2}$ & 50 & $<1$ & $<1$ \\
4 & $\mathrm{ZnMe}_{2}$ & 50 & 95 & 3 \\
5 & $\mathrm{ZnMe}_{2}$ & 80 & $95^{d}$ & $<1$ \\
6 & $\mathrm{ZnPh}_{2}$ & 50 & $96(88)^{e}$ & 3 \\
7 & $\mathrm{ZnCl}_{2}$ & 50 & $<1$ & $<1$ \\
$8^{b}$ & $\mathrm{AlMe}_{3}$ & 50 & $<1$ & $<1$ \\
$9^{b, c}$ & $\mathrm{AlMe}_{3}$ & 50 & $<1$ & $<1$ \\
10 & $\mathrm{AlMe}_{3}$ & 50 & 5 & $82(80)^{e}$ \\
11 & $\mathrm{AlMe}_{3}$ & 80 & 17 & $56^{f}$ \\
12 & $\mathrm{AlMe}_{2} \mathrm{Cl}$ & 50 & 2 & 34 \\
13 & $\mathrm{AlMeCl}_{2}$ & 50 & $<1$ & 13 \\
14 & $\mathrm{AlCl}_{3}$ & 50 & $<1$ & 6 \\
\hline
\end{tabular}

${ }^{a}$ Determined by GC based on $\mathbf{2 a} .{ }^{b}$ Reaction runs in the absence of Ni(cod) ${ }_{2} \cdot{ }^{c}$ Reaction runs in the absence of $\mathrm{P}(i-\operatorname{Pr})_{3} \cdot{ }^{d} E / Z=93: 7 .{ }^{e}$ Isolated yields based on $2 \mathbf{a} .{ }^{f} Z E / E E /$ others $=52: 43: 5$.

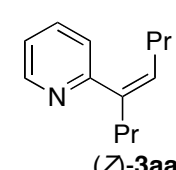

(Z)-2-(4-Octen-4-yl)pyridine $\quad[(Z)-3 a a]$. A colorless oil, $\mathrm{R}_{\mathrm{f}} 0.50$ (hexane-ethyl acetate $=$ 9:1). ${ }^{1} \mathrm{H}$ NMR $\left(400 \mathrm{MHz}, \mathrm{CDCl}_{3}\right) \delta 8.54$ (ddd, $\left.J=4.8,1.6,0.7 \mathrm{~Hz}, 1 \mathrm{H}\right), 7.53$ (td, $J=7.6$, $0.9 \mathrm{~Hz}, 1 \mathrm{H}), 7.11-7.00(\mathrm{~m}, 2 \mathrm{H}), 5.51(\mathrm{t}, J=7.3 \mathrm{~Hz}, 1 \mathrm{H}), 2.39(\mathrm{t}, J=7.5 \mathrm{~Hz}, 2 \mathrm{H}), 1.93(\mathrm{q}, J$ $(\mathrm{z})$-3аa $=7.3 \mathrm{~Hz}, 2 \mathrm{H}), 1.38-1.20(\mathrm{~m}, 4 \mathrm{H}), 0.81(\mathrm{t}, J=7.3 \mathrm{~Hz}, 3 \mathrm{H}), 0.78(\mathrm{t}, J=7.4 \mathrm{~Hz}, 3 \mathrm{H}) ;{ }^{13} \mathrm{C}$ NMR (101 MHz, $\left.\mathrm{CDCl}_{3}\right) \delta 159.5,148.9,140.1,135.2,129.0,123.6,120.9,39.6,30.8,23.1,21.3,13.7$, 13.6; Anal. Calcd for $\mathrm{C}_{13} \mathrm{H}_{19} \mathrm{~N}$ : C, 82.48; H, 10.12. Found: C, 82.23; H, 10.11 .

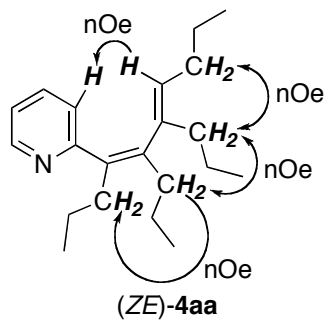

(4Z,6E)-5,6-Dipropyl-4-(2-pyridyl)deca-4,6-diene [(ZE)-4aa]. A colorless oil, $\mathrm{R}_{\mathrm{f}} 0.75$ (hexane-ethyl acetate $\left.=9: 1\right) .{ }^{1} \mathrm{H} \mathrm{NMR}\left(400 \mathrm{MHz}, \mathrm{CDCl}_{3}\right) \delta 8.50(\mathrm{dq}, J=$ $4.8,1.0 \mathrm{~Hz}, 1 \mathrm{H}), 7.44(\mathrm{td}, J=7.6,1.9 \mathrm{~Hz}, 1 \mathrm{H}), 7.03-6.96(\mathrm{~m}, 2 \mathrm{H}), 4.91(\mathrm{t}, J=7.3$ $\mathrm{Hz}, 1 \mathrm{H}), 2.53(\mathrm{t}, J=7.8 \mathrm{~Hz}, 2 \mathrm{H}), 2.23(\mathrm{t}, J=7.8 \mathrm{~Hz}, 2 \mathrm{H}), 1.91(\mathrm{t}, J=8.2 \mathrm{~Hz}, 2 \mathrm{H})$, $1.73(\mathrm{q}, J=7.3 \mathrm{~Hz}, 2 \mathrm{H}), 1.47-1.20(\mathrm{~m}, 6 \mathrm{H}), 1.04-0.78(\mathrm{~m}, 11 \mathrm{H}), 0.65(J=7.3 \mathrm{~Hz}$, $3 \mathrm{H}) ;{ }^{13} \mathrm{C}$ NMR $\left(101 \mathrm{MHz}, \mathrm{CDCl}_{3}\right) \delta 162.8,148.2,142.7,138.9,136.4,134.7$, $132.0,125.1,120.1,34.7,33.0,32.6,30.9,22.6,22.3,21.7,21.6,14.9,14.3,14.1$, 13.9; HRMS (EI) Calcd for $\mathrm{C}_{21} \mathrm{H}_{33} \mathrm{~N}$ : 299.2613. Found: $m / z$ 299.2602. The assignments were made based on HMQC, HMBC, and NOE experiments. 


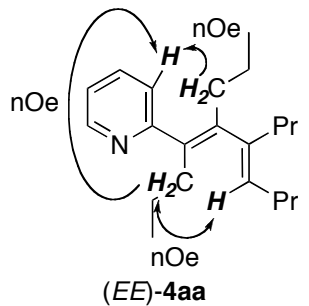

(4E,6E)-5,6-Dipropyl-4-(2-pyridyl)deca-4,6-diene [(EE)-4aa]. A colorless oil, $\mathrm{R}_{\mathrm{f}}$ 0.40 (hexane-ethyl acetate = 9:1). ${ }^{1} \mathrm{H}$ NMR $\left(400 \mathrm{MHz}, \mathrm{CDCl}_{3}\right) \delta 8.61(\mathrm{dt}, J=4.6$, $1.3 \mathrm{~Hz}, 1 \mathrm{H}), 7.60(\mathrm{td}, J=6.6,1.9 \mathrm{~Hz}, 1 \mathrm{H}), 7.13-7.07(\mathrm{~m}, 2 \mathrm{H}), 5.24(\mathrm{t}, J=7.3 \mathrm{~Hz}$, $1 \mathrm{H}), 2.47(\mathrm{t}, J=7.9 \mathrm{~Hz}, 2 \mathrm{H}), 2.16-2.07(\mathrm{~m}, 4 \mathrm{H}), 1.84(\mathrm{t}, J=7.8 \mathrm{~Hz}, 2 \mathrm{H})$, $1.51-1.39(\mathrm{~m}, 4 \mathrm{H}), 1.26(\mathrm{sext}, J=7.5 \mathrm{~Hz}, 2 \mathrm{H}), 1.14(\mathrm{sext}, J=7.6 \mathrm{~Hz}, 2 \mathrm{H}), 0.96(\mathrm{t}$, $J=7.3 \mathrm{~Hz}, 3 \mathrm{H}), 0.94(\mathrm{t}, J=7.3 \mathrm{~Hz}, 3 \mathrm{H}), 0.78(\mathrm{t}, J=7.3 \mathrm{~Hz}, 3 \mathrm{H}), 0.71(\mathrm{t}, J=7.3$ $\mathrm{Hz}, 3 \mathrm{H}) ;{ }^{13} \mathrm{C}$ NMR $\left(101 \mathrm{MHz}, \mathrm{CDCl}_{3}\right) \delta 161.8,149.1,141.6,138.5,138.8,135.3$, 128.5, 124.2, 120.7, 36.5, 33.5, 31.8, 30.0, 23.3, 22.3, 21.8, 21.7, 14.8, 14.4, 14.10, 14.06; HRMS (EI) Calcd for $\mathrm{C}_{21} \mathrm{H}_{33} \mathrm{~N}$ : 299.2613. Found: $\mathrm{m} / z$ 299.2621. The assignments were made based on HMQC, $\mathrm{HMBC}$, and NOE experiments.

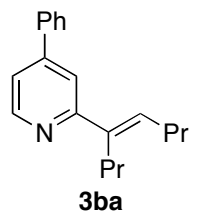

(E)-2-(4-Octen-4-yl)-4-phenylpyridine (3ba). A colorless oil, $\mathrm{R}_{\mathrm{f}} 0.30$ (hexane-ethyl acetate $=9: 1) .{ }^{1} \mathrm{H}$ NMR $\left(400 \mathrm{MHz}, \mathrm{CDCl}_{3}\right) \delta 8.61(\mathrm{dd}, J=5.1,0.7 \mathrm{~Hz}, 1 \mathrm{H}), 7.69-7.61$ $(\mathrm{m}, 2 \mathrm{H}), 7.58(\mathrm{~d}, J=1.9 \mathrm{~Hz}, 1 \mathrm{H}), 7.52-7.40(\mathrm{~m}, 3 \mathrm{H}), 7.32(\mathrm{dd}, J=5.1,1.8 \mathrm{~Hz}, 1 \mathrm{H}), 6.30$ $(\mathrm{t}, J=7.4 \mathrm{~Hz}, 1 \mathrm{H}), 2.70(\mathrm{t}, J=7.7 \mathrm{~Hz}, 2 \mathrm{H}), 2.29(\mathrm{q}, J=7.4 \mathrm{~Hz}, 2 \mathrm{H}), 1.56(\mathrm{sext}, J=7.4$ $\mathrm{Hz}, 2 \mathrm{H}), 1.49$ (sext, $J=7.5 \mathrm{~Hz}, 2 \mathrm{H}), 1.02(\mathrm{t}, J=7.4 \mathrm{~Hz}, 3 \mathrm{H}), 0.97$ (t, $J=7.3 \mathrm{~Hz}, 3 \mathrm{H})$; ${ }^{13} \mathrm{C}$ NMR $\left(101 \mathrm{MHz}, \mathrm{CDCl}_{3}\right) \delta 160.4,149.0,148.4,139.7,138.6,132.2,128.8,128.6,126.8,119.2$, 118.3, 30.9, 30.3, 22.9, 22.2, 14.22, 14.17; Anal. Calcd for $\mathrm{C}_{19} \mathrm{H}_{23} \mathrm{~N}$ : C, 85.99; H, 8.74. Found: C, $85.90 ; \mathrm{H}, 8.80$.

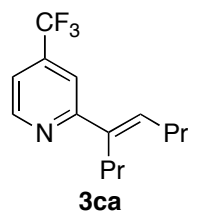

(E)-2-(4-Octen-4-yl)-4-(trifluoromethyl)pyridine (3ca). A colorless oil, $\mathrm{R}_{\mathrm{f}} 0.60$ (hexane-ethyl acetate $=9: 1) .{ }^{1} \mathrm{H}$ NMR $\left(400 \mathrm{MHz}, \mathrm{CDCl}_{3}\right) \delta 8.71(\mathrm{~d}, J=4.9 \mathrm{~Hz}, 1 \mathrm{H}), 7.56$ $(\mathrm{t}, J=0.8 \mathrm{~Hz}, 1 \mathrm{H}), 7.30(\mathrm{dd}, J=5.0,1.0 \mathrm{~Hz}, 1 \mathrm{H}), 6.30(\mathrm{t}, J=7.5 \mathrm{~Hz}, 1 \mathrm{H}), 2.64(\mathrm{t}, J=7.7$ $\mathrm{Hz}, 2 \mathrm{H}), 2.27(\mathrm{q}, J=7.4 \mathrm{~Hz}, 2 \mathrm{H}), 1.53$ (sext, $J=7.5 \mathrm{~Hz}, 2 \mathrm{H}), 1.43$ (sext, $J=7.5 \mathrm{~Hz}, 2 \mathrm{H}$ ), $0.99(\mathrm{t}, J=7.4 \mathrm{~Hz}, 3 \mathrm{H}), 0.93(\mathrm{t}, J=7.4 \mathrm{~Hz}, 3 \mathrm{H}) ;{ }^{13} \mathrm{C} \mathrm{NMR}\left(101 \mathrm{MHz}, \mathrm{CDCl}_{3}\right) \delta 161.2$, $149.5,139.0,138.2(\mathrm{q}, J=33.2 \mathrm{~Hz}), 134.1,123.0(\mathrm{q}, J=272.4 \mathrm{~Hz}), 116.4(\mathrm{q}, J=3.3 \mathrm{~Hz}), 115.6(\mathrm{q}, J=$ 3.6 Hz), 31.0, 30.1, 22.8, 22.1, 14.21, 14.18; 19F NMR (282 MHz, $\left.\mathrm{CDCl}_{3}\right) \delta-65.3$; Anal. Calcd for $\mathrm{C}_{14} \mathrm{H}_{18} \mathrm{~F}_{3} \mathrm{~N}$ : C, 82.48; H, 10.12. Found: C, 82.20; H, 10.04 .

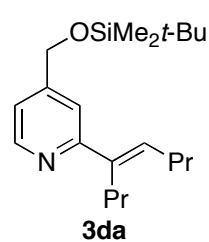

(E)-4-[(tert-Butyldimethylsilyloxy)methyl]-2-(4-octen-4-yl)pyridine (3da). A colorless oil, $\mathrm{R}_{\mathrm{f}} 0.50$ (hexane-ethyl acetate $\left.=9: 1\right) .{ }^{1} \mathrm{H}$ NMR $\left(400 \mathrm{MHz}, \mathrm{CDCl}_{3}\right) \delta 8.48(\mathrm{~d}, J=5.1$ $\mathrm{Hz}, 1 \mathrm{H}), 7.34(\mathrm{~s}, 1 \mathrm{H}), 7.04(\mathrm{dd}, J=5.0,0.6 \mathrm{~Hz}, 1 \mathrm{H}), 6.24(\mathrm{t}, J=7.3 \mathrm{~Hz}, 1 \mathrm{H}), 4.73(\mathrm{~s}$, $2 \mathrm{H}), 2.60(\mathrm{t}, J=7.7 \mathrm{~Hz}, 2 \mathrm{H}), 2.24(\mathrm{q}, J=7.4 \mathrm{~Hz}, 2 \mathrm{H}), 1.51$ (sext, $J=7.4 \mathrm{~Hz}, 2 \mathrm{H}), 1.42$ (sext, $J=7.3 \mathrm{~Hz}, 2 \mathrm{H}), 0.98(\mathrm{t}, J=7.4 \mathrm{~Hz}, 3 \mathrm{H}), 0.97(\mathrm{~s}, 9 \mathrm{H}), 0.92(\mathrm{t}, J=7.2 \mathrm{~Hz}, 3 \mathrm{H}), 0.12$ $(\mathrm{s}, 6 \mathrm{H}) ;{ }^{13} \mathrm{C} \mathrm{NMR}\left(101 \mathrm{MHz}, \mathrm{CDCl}_{3}\right) \delta 159.7,150.4,148.5,139.5,132.0,118.2,117.0$, 63.7, 30.9, 30.3, 26.0, 23.0, 22.2, 18.5, 14.25, 14.18, -5.2; Anal. Calcd for $\mathrm{C}_{20} \mathrm{H}_{35} \mathrm{NOSi}$ : C, 72.01; $\mathrm{H}$, 10.58. Found: C, 72.26; H, 10.44 .

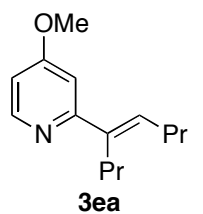

(E)-4-Methoxy-2-(4-octen-4-yl)pyridine (3ea). A colorless oil, $\mathrm{R}_{\mathrm{f}} 0.60$ (hexane-ethyl acetate $=1: 1) .{ }^{1} \mathrm{H}$ NMR $\left(400 \mathrm{MHz}, \mathrm{CDCl}_{3}\right) \delta 8.37(\mathrm{~d}, J=5.6 \mathrm{~Hz}, 1 \mathrm{H}), 6.86(\mathrm{~d}, J=2.6 \mathrm{~Hz}$, $1 \mathrm{H}), 6.64(\mathrm{dd}, J=5.7,2.4 \mathrm{~Hz}, 1 \mathrm{H}), 6.19(\mathrm{t}, J=7.4 \mathrm{~Hz}, 1 \mathrm{H}), 3.89(\mathrm{~s}, 3 \mathrm{H}), 2.57(\mathrm{t}, J=7.7$ $\mathrm{Hz}, 2 \mathrm{H}), 2.22(\mathrm{q}, J=7.4 \mathrm{~Hz}, 2 \mathrm{H}), 1.49$ (sext, $J=7.4 \mathrm{~Hz}, 2 \mathrm{H}), 1.40(\mathrm{sext}, J=7.3 \mathrm{~Hz}, 2 \mathrm{H})$, $0.97(\mathrm{t}, J=7.3 \mathrm{~Hz}, 3 \mathrm{H}), 0.91(\mathrm{t}, J=7.4 \mathrm{~Hz}, 3 \mathrm{H}) ;{ }^{13} \mathrm{C} \mathrm{NMR}\left(101 \mathrm{MHz}, \mathrm{CDCl}_{3}\right) \delta 165.8$, 161.6, 149.8, 139.5, 132.0, 107.0, 106.6, 55.0, 30.8, 30.4, 22.9, 22.1, 14.21, 14.17; HRMS (EI) Calcd for $\mathrm{C}_{14} \mathrm{H}_{21} \mathrm{NO}: 219.1623$. Found: $m / z$ 219.1614. 


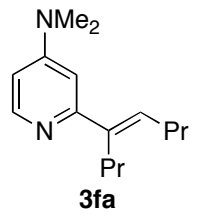

4-Dimethylamino-2-(4-octen-4-yl)pyridine (3fa, $E / Z=97: 3)$. A colorless oil, $\mathrm{R}_{\mathrm{f}} 0.70$ (hexane-ethyl acetate $=1: 1) .{ }^{1} \mathrm{H}$ NMR $\left(400 \mathrm{MHz}, \mathrm{CDCl}_{3}\right) \delta 8.22(\mathrm{dd}, J=5.8,0.7 \mathrm{~Hz}$, $0.03 \mathrm{H}), 8.18(\mathrm{~d}, J=6.0 \mathrm{~Hz}, 0.97 \mathrm{H}), 6.54(\mathrm{~d}, J=2.6 \mathrm{~Hz}, 1 \mathrm{H}), 6.35(\mathrm{dd}, J=6.1,2.6 \mathrm{~Hz}$, $1 \mathrm{H}), 6.08(\mathrm{t}, J=7.4 \mathrm{~Hz}, 0.97 \mathrm{H}), 5.48(\mathrm{t}, J=7.5 \mathrm{~Hz}, 0.03 \mathrm{H}), 2.99(\mathrm{~s}, 5.82 \mathrm{H}), 2.98(\mathrm{~s}, 0.18$ $\mathrm{H}), 2.57(\mathrm{t}, J=7.6 \mathrm{~Hz}, 1.94 \mathrm{H}), 2.40(\mathrm{t}, J=7.3 \mathrm{~Hz}, 0.06 \mathrm{H}), 2.19(\mathrm{q}, J=7.3 \mathrm{~Hz}, 1.94 \mathrm{H})$, $2.01(\mathrm{q}, J=7.4 \mathrm{~Hz}, 0.06 \mathrm{H}), 1.55-1.31(\mathrm{~m}, 4 \mathrm{H}), 1.01-0.84(\mathrm{~m}, 6 \mathrm{H}) ;{ }^{13} \mathrm{C} \mathrm{NMR}$ [for $(E)-3 g a, 101 \mathrm{MHz}$, $\left.\mathrm{CDCl}_{3}\right] \delta 160.4,154.6,148.6,140.6,130.8,104.6,103.3,39.2,30.8,30.6,23.0,22.1,14.23,14.17$; Anal. Calcd for $\mathrm{C}_{15} \mathrm{H}_{24} \mathrm{~N}_{2}$; HRMS (EI) Calcd for $\mathrm{C}_{15} \mathrm{H}_{24} \mathrm{~N}_{2}$ : 232.1939. Found: $m / z$ 232.1945.

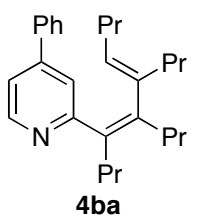

(4Z,6E)-5,6-Dipropyl-4-[2-(4-phenylpyridin-2-yl)]deca-4,6-diene (4ba). A colorless oil, $\mathrm{R}_{\mathrm{f}} 0.35$ (hexane-ethyl acetate $\left.=9: 1\right) .{ }^{1} \mathrm{H} \mathrm{NMR}\left(400 \mathrm{MHz}, \mathrm{CDCl}_{3}\right) \delta 8.55(\mathrm{dd}, J=5.2$, $0.8 \mathrm{~Hz}, 1 \mathrm{H}), 7.58-7.52(\mathrm{~m}, 2 \mathrm{H}), 7.48-7.37(\mathrm{~m}, 3 \mathrm{H}), 7.25(\mathrm{~s}, 1 \mathrm{H}), 7.22(\mathrm{dd}, J=5.1,1.8 \mathrm{~Hz}$, $1 \mathrm{H}), 4.99(\mathrm{t}, J=7.3 \mathrm{~Hz}, 1 \mathrm{H}), 2.57(\mathrm{t}, J=7.7 \mathrm{~Hz}, 2 \mathrm{H}), 2.26(\mathrm{t}, J=7.8 \mathrm{~Hz}, 2 \mathrm{H}), 1.94(\mathrm{t}, J=$ $8.2 \mathrm{~Hz}, 2 \mathrm{H}), 1.75(\mathrm{q}, J=7.3 \mathrm{~Hz}, 2 \mathrm{H}), 1.48-1.24(\mathrm{~m}, 6 \mathrm{H}), 1.01-0.83(\mathrm{~m}, 11 \mathrm{H}), 0.59(\mathrm{t}, J=$ $7.4 \mathrm{~Hz}, 3 \mathrm{H}) ;{ }^{13} \mathrm{C}$ NMR $\left(101 \mathrm{MHz}, \mathrm{CDCl}_{3}\right) \delta 163.3,148.8,147.1,142.9,139.1,138.7,136.6,132.1$, 128.8, 128.5, 126.8, 123.2, 118.3, 34.7, 33.2, 32.8, 30.1, 22.7, 22.5, 21.8, 21.7, 14.9, 14.3, 14.2, 13.8; HRMS (EI) Calcd for $\mathrm{C}_{27} \mathrm{H}_{37} \mathrm{~N}: 375.2926$. Found: $m / z$ 375.2922.

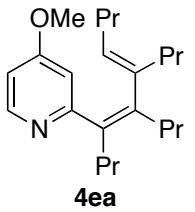

(4Z,6E)-5,6-Dipropyl-4-[2-(4-methoxypyridin-2-yl)]deca-4,6-diene (4ea). A colorless oil, $\mathrm{R}_{\mathrm{f}} 0.75$ (hexane-ethyl acetate $\left.=1: 1\right) .{ }^{1} \mathrm{H} \mathrm{NMR}\left(400 \mathrm{MHz}, \mathrm{CDCl}_{3}\right) \delta 8.32(\mathrm{dd}, J=5.5$, $0.7 \mathrm{~Hz}, 1 \mathrm{H}), 6.58-6.53(\mathrm{~m}, 2 \mathrm{H}), 4.95(\mathrm{t}, J=7.4 \mathrm{~Hz}, 1 \mathrm{H}), 3.77(\mathrm{~s}, 3 \mathrm{H}), 2.50(\mathrm{t}, J=7.8 \mathrm{~Hz}$, $2 \mathrm{H}), 2.22(\mathrm{t}, J=7.8 \mathrm{~Hz}, 2 \mathrm{H}), 1.92(\mathrm{t}, J=8.3 \mathrm{~Hz}, 2 \mathrm{H}), 1.76(\mathrm{q}, J=7.3 \mathrm{~Hz}, 2 \mathrm{H}), 1.46-1.20$ $(\mathrm{m}, 6 \mathrm{H}), 1.08-0.75(\mathrm{~m}, 11 \mathrm{H}), 0.66(\mathrm{t}, J=7.3 \mathrm{~Hz}, 3 \mathrm{H}) ;{ }^{13} \mathrm{C} \mathrm{NMR}\left(101 \mathrm{MHz}, \mathrm{CDCl}_{3}\right) \delta$ 164.7, 164.3, 149.4, 142.4, 139.0, 136.5, 131.5, 110.8, 106.8, 54.9, 34.6, 33.1, 32.6, 30.1, 22.7, 22.4, 21.71, 21.65, 14.9, 14.3, 14.1, 13.9; HRMS (EI) Calcd for $\mathrm{C}_{22} \mathrm{H}_{35} \mathrm{NO}: 329.2719$. Found: $\mathrm{m} / z$ 329.2716.

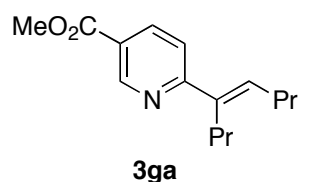

Methyl (E)-6-(4-octen-4-yl)nicotinate (3ga). A colorless oil, $\mathrm{R}_{\mathrm{f}} \quad 0.70$ (hexane-ethyl acetate =9:1). ${ }^{1} \mathrm{H}$ NMR $\left(400 \mathrm{MHz}, \mathrm{CDCl}_{3}\right) \delta 9.12(\mathrm{q}, J=1.0 \mathrm{~Hz}$, $1 \mathrm{H}), 8.17(\mathrm{dt}, J=8.4,1.1 \mathrm{~Hz}, 1 \mathrm{H}), 7.42(\mathrm{dd}, J=8.3,0.8 \mathrm{~Hz}, 1 \mathrm{H}), 6.40(\mathrm{t}, J=7.3$ $\mathrm{Hz}, 1 \mathrm{H}), 3.93(\mathrm{~s}, 3 \mathrm{H}), 2.62(\mathrm{t}, J=7.7 \mathrm{~Hz}, 2 \mathrm{H}), 2.60$ (q, $J=7.4 \mathrm{~Hz}, 2 \mathrm{H}), 1.59-1.21$ $(\mathrm{m}, 4 \mathrm{H}), 1.04-0.81(\mathrm{~m}, 6 \mathrm{H}) ;{ }^{13} \mathrm{C} \mathrm{NMR}\left(101 \mathrm{MHz}, \mathrm{CDCl}_{3}\right) \delta 165.8,163.3,150.0$, 139.1, 137.1, 134.9, 123.1, 119.4, 52.2, 31.0, 30.0, 22.8, 22.2, 14.20, 14.17; Anal. Calcd for $\mathrm{C}_{15} \mathrm{H}_{21} \mathrm{NO}_{2}$ : C, 72.84; H, 8.56. Found: C, 72.82; H, 8.47.

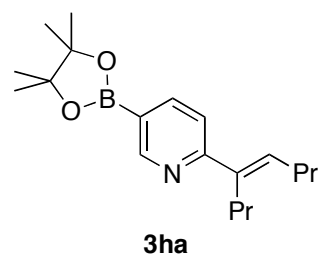

2-(4-Octen-4-yl)-5-(4,4,5,5-tetramethyl-1,3,2-dioxaborolan-2-yl)pyridine (3ha, $\boldsymbol{E} / \boldsymbol{Z}=98: 2)$. A colorless oil, $\mathrm{R}_{\mathrm{f}} 0.70$ (hexane-ethyl acetate $\left.=9: 1\right) .{ }^{1} \mathrm{H}$ NMR (400 $\left.\mathrm{MHz} \mathrm{CDCl}_{3}\right) \delta 8.94(\mathrm{q}, J=0.9 \mathrm{~Hz}, 0.02 \mathrm{H}), 8.87(\mathrm{q}, J=0.9 \mathrm{~Hz}, 0.98 \mathrm{H}), 8.00(\mathrm{dd}$, $J=7.8,1.9 \mathrm{~Hz}, 0.02 \mathrm{H}), 7.97(\mathrm{dd}, J=7.8,1.8 \mathrm{~Hz}, 0.98 \mathrm{H}), 7.34(\mathrm{dd}, J=7.8,0.9 \mathrm{~Hz}$, $0.98 \mathrm{H}), 7.13(\mathrm{dd}, J=7.8,1.0 \mathrm{~Hz}, 0.02 \mathrm{H}), 6.27(\mathrm{t}, J=7.4 \mathrm{~Hz}, 0.98 \mathrm{H}), 5.58(\mathrm{t}, J$ $=7.4 \mathrm{~Hz}, 0.02 \mathrm{H}), 2.62(\mathrm{t}, J=7.6 \mathrm{~Hz}, 1.96 \mathrm{H}), 2.44(\mathrm{t}, J=7.5 \mathrm{~Hz}, 0.04 \mathrm{H}), 2.24(\mathrm{q}, J$ $=7.4 \mathrm{~Hz}, 1.96 \mathrm{H}), 1.98(\mathrm{q}, J=7.5 \mathrm{~Hz}, 0.04 \mathrm{H}), 1.56-1.17(\mathrm{~m}, 16 \mathrm{H}), 1.02-0.80(\mathrm{~m}, 6 \mathrm{H}) ;{ }^{13} \mathrm{C}$ NMR [for (E)-3ga, $\left.101 \mathrm{MHz}, \mathrm{CDCl}_{3}\right] \delta$ 161.7, 154.3, 142.6, 139.4, 133.5, 119.6, 84.0, 31.0, 30.2, 24.9, 22.9, 22.1, 14.21, 14.19; Anal. Calcd for $\mathrm{C}_{19} \mathrm{H}_{30} \mathrm{BNO}_{2}$ : C, 72.39; H, 9.59. Found: C, 72.35; H, 9.66.

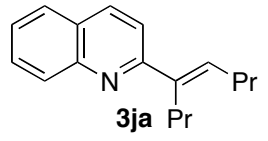

(E)-2-(4-Octen-4-yl)quinoline (3ja). A colorless oil, $\mathrm{R}_{\mathrm{f}} 0.50$ (hexane-ethyl acetate $=$ 9:1). ${ }^{1} \mathrm{H}$ NMR $\left(400 \mathrm{MHz}, \mathrm{CDCl}_{3}\right) \delta 8.05(\mathrm{~d}, J=8.7 \mathrm{~Hz}, 2 \mathrm{H}), 7.75(\mathrm{dd}, J=8.2,1.6 \mathrm{~Hz}$, $1 \mathrm{H}), 7.66(\mathrm{ddd}, J=8.3,7.0,1.4 \mathrm{~Hz}, 1 \mathrm{H}), 7.56(\mathrm{~d}, J=8.6 \mathrm{~Hz}, 1 \mathrm{H}), 7.46(\mathrm{ddd}, J=8.1$, 
6.9, $1.2 \mathrm{~Hz}, 1 \mathrm{H}), 6.32(\mathrm{t}, J=7.4 \mathrm{~Hz}, 1 \mathrm{H}), 2.79(\mathrm{t}, J=7.7,2 \mathrm{H}), 2.32(\mathrm{t}, J=7.4 \mathrm{~Hz}, 2 \mathrm{H}), 1.62-1.40(\mathrm{~m}$, $4 \mathrm{H}), 1.01(\mathrm{t}, J=7.3 \mathrm{~Hz}, 3 \mathrm{H}), 0.95(\mathrm{t}, J=7.3 \mathrm{~Hz}, 3 \mathrm{H}) ;{ }^{13} \mathrm{C} \mathrm{NMR}\left(101 \mathrm{MHz}, \mathrm{CDCl}_{3}\right) \delta 159.8,147.5$, 140.6, 135.7, 133.7, 129.3, 129.0, 127.1, 126.7, 125.5, 119.1, 31.1, 30.4, 22.9, 22.4, 14.3, 14.2; Anal. Calcd for $\mathrm{C}_{17} \mathrm{H}_{21} \mathrm{~N}$ : HRMS (EI) Calcd for $\mathrm{C}_{17} \mathrm{H}_{21} \mathrm{~N}$ : 239.1674. Found: $m / z 239.1679$.

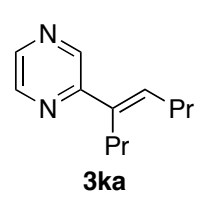

2-(4-Octen-4-yl)pyrazine (3ka, $\boldsymbol{E} / \boldsymbol{Z}=\mathbf{9 9 : 1 )}$. A colorless oil, $\mathrm{R}_{\mathrm{f}} 0.30$ (hexane-ethyl acetate $=9: 1) .{ }^{1} \mathrm{H}$ NMR $\left(400 \mathrm{MHz}, \mathrm{CDCl}_{3}\right) \delta 8.88(\mathrm{~d}, J=1.5 \mathrm{~Hz}, 0.01 \mathrm{H}), 8.66(\mathrm{~d}, J=1.5$ $\mathrm{Hz}, 0.99 \mathrm{H}), 8.56(\mathrm{dd}, J=1.3 \mathrm{~Hz}, 0.01 \mathrm{H}), 8.46(\mathrm{dd}, J=2.5,1.6 \mathrm{~Hz}, 0.99 \mathrm{H}), 8.39(\mathrm{~d}, J=$ $2.4 \mathrm{~Hz}, 0.01 \mathrm{H}), 8.34(\mathrm{~d}, J=2.5 \mathrm{~Hz}, 0.99 \mathrm{H}), 6.28(\mathrm{t}, J=7.3 \mathrm{~Hz}, 0.99 \mathrm{H}), 5.71(\mathrm{t}, J=7.4 \mathrm{~Hz}$, $0.01 \mathrm{H}), 2.61(\mathrm{t}, J=7.7 \mathrm{~Hz}, 1.98 \mathrm{H}), 2.46(\mathrm{t}, J=7.0 \mathrm{~Hz}, 0.02 \mathrm{H}), 2.26(\mathrm{q}, J=7.4 \mathrm{~Hz}$, $1.98 \mathrm{H}), 2.03(\mathrm{q}, J=7.4 \mathrm{~Hz}, 0.02 \mathrm{H}), 1.58-1.31(\mathrm{~m}, 4 \mathrm{H}), 1.03-0.80(\mathrm{~m}, 6 \mathrm{H}) ;{ }^{13} \mathrm{C}$ NMR [for $(E)-3 \mathrm{ka}, 101$ $\left.\mathrm{MHz}, \mathrm{CDCl}_{3}\right] \delta$ 155.2, 143.1, 142.0, 141.7, 137.1, 134.3, 30.9, 29.8, 22.8, 22.1, 14.2, 14.1; Anal. Calcd for $\mathrm{C}_{12} \mathrm{H}_{18} \mathrm{~N}_{2}$ : C, 75.74; H, 9.53. Found: C, 76.04; H, 9.76.

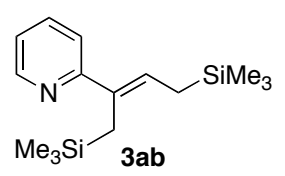

(Z)-2-(1,4-Bis(trimethylsilyl)-2-buten-2-yl)pyridine (3ab). A colorless oil, $\mathrm{R}_{\mathrm{f}} 0.45$ (hexane-ethyl acetate $=9: 1) .{ }^{1} \mathrm{H} \mathrm{NMR}\left(400 \mathrm{MHz} \mathrm{CDCl}_{3}\right) \delta 8.50(\mathrm{dt}, J=4.9,0.9 \mathrm{~Hz}$, $1 \mathrm{H}), 7.56(\mathrm{td}, J=7.8,1.8 \mathrm{~Hz}, 1 \mathrm{H}), 7.35(\mathrm{dd}, J=8.0,0.9,1 \mathrm{H}), 7.05(\mathrm{dd}, J=7.4,4.9$ $\mathrm{Hz}, 1 \mathrm{H}), 6.09(\mathrm{t}, J=8.7 \mathrm{~Hz}, 1 \mathrm{H}), 2.14(\mathrm{~s}, 2 \mathrm{H}), 1.63(\mathrm{~d}, J=8.6 \mathrm{~Hz}, 2 \mathrm{H}), 0.06(\mathrm{~d}, J=$ $0.6,9 \mathrm{H}),-0.12(\mathrm{~s}, 9 \mathrm{H}) ;{ }^{13} \mathrm{C} \mathrm{NMR}\left(101 \mathrm{MHz}, \mathrm{CDCl}_{3}\right) \delta 161.1,148.2,135.7,124.9,120.5,120.0,20.8$, $18.5,-0.7,-1.3$; HRMS (FAB+) Calcd for $\mathrm{C}_{15} \mathrm{H}_{27} \mathrm{NSi}_{2}$ : 277.1682. Found: $m / z$ 277.1684.

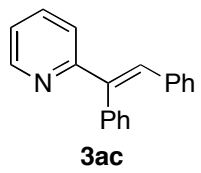

(E)-2-(1,2-diphenylvinyl)pyridine (3ac). A colorless oil, $\mathrm{R}_{\mathrm{f}} 0.50$ (hexane-ethyl acetate $=$ 9:1). ${ }^{1} \mathrm{H}$ NMR $\left(400 \mathrm{MHz}, \mathrm{CDCl}_{3}\right) \delta 8.67(\mathrm{~d}, J=4.1 \mathrm{~Hz}, 1 \mathrm{H}), 7.89(\mathrm{~s}, 1 \mathrm{H}), 7.57$ (td, $J=$ $7.8,1.8 \mathrm{~Hz}, 1 \mathrm{H}), 7.46-7.01(\mathrm{~m}, 11 \mathrm{H}), 6.98(\mathrm{~d}, \mathrm{~J}=8.1 \mathrm{~Hz}, 1 \mathrm{H}) ;{ }^{13} \mathrm{C}$ NMR $(101 \mathrm{MHz}$, $\left.\mathrm{CDCl}_{3}\right) \delta 158.7,149.0,140.3,139.0,136.6,136.2,130.7,130.1,129.9,128.9,127.8$, 127.5, 127.1, 122.3, 121.8; HRMS (FAB+) Calcd for $\mathrm{C}_{19} \mathrm{H}_{15} \mathrm{~N}: 257.1204$. Found: $m / z$ 257.1201.

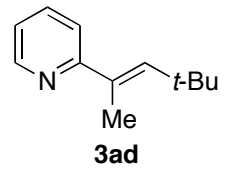

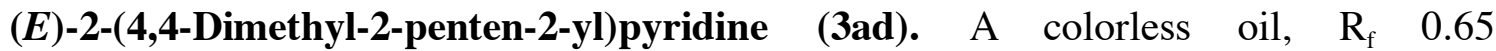
(hexane-ethyl acetate $=1: 1) .{ }^{1} \mathrm{H} \mathrm{NMR}\left(400 \mathrm{MHz}, \mathrm{CDCl}_{3}\right) \delta 8.53$ (ddd, $J=4.8,1.9,0.8$ $\mathrm{Hz}, 1 \mathrm{H}), 7.58(\mathrm{td}, J=7.7,1.9 \mathrm{~Hz}, 1 \mathrm{H}), 7.35(\mathrm{dt}, J=8.0,1.0 \mathrm{~Hz}, 1 \mathrm{H}), 7.07$ (ddd, $J=7.5$, $4.8,1.0 \mathrm{~Hz}, 1 \mathrm{H}), 6.31(\mathrm{q}, J=1.3 \mathrm{~Hz}, 1 \mathrm{H}), 2.22(\mathrm{~d}, J=1.3 \mathrm{~Hz}, 3 \mathrm{H}), 1.25(\mathrm{~s}, 9 \mathrm{H}) ;{ }^{13} \mathrm{C}$ $\operatorname{NMR}\left(101 \mathrm{MHz}, \mathrm{CDCl}_{3}\right) \delta 161.6,148.3,141.8,136.0,133.8,121.0,119.7,32.8,30.8,15.6$; Anal. Calcd for $\mathrm{C}_{12} \mathrm{H}_{17} \mathrm{~N}$ : C, 82.23; H, 9.78. Found: C, 82.46; H, 9.99.

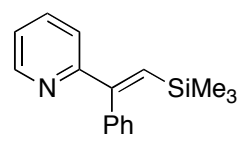

$(\boldsymbol{E})$-2-[1-Phenyl-2-(trimethylsilyl)ethenyl]pyridine [(E)-3ae]. A colorless oil, $\mathrm{R}_{\mathrm{f}} 0.30$ (hexane-ethyl acetate $=9: 1) .{ }^{1} \mathrm{H} \mathrm{NMR}\left(400 \mathrm{MHz}, \mathrm{CDCl}_{3}\right) \delta 8.63(\mathrm{ddd}, J=4.8,1.8,0.9$ $\mathrm{Hz}, 1 \mathrm{H}), 7.52(\mathrm{td}, J=7.6,1.8,1 \mathrm{H}), 7.42-7.31(\mathrm{~m}, 3 \mathrm{H}), 7.30-7.20(\mathrm{~m}, 2 \mathrm{H}), 7.17(\mathrm{~s}, 1 \mathrm{H})$, $7.13(\mathrm{ddd}, J=7.5,4.7,1.1 \mathrm{~Hz}, 1 \mathrm{H}), 6.93(\mathrm{dt}, J=8.1,1.1 \mathrm{~Hz}, 1 \mathrm{H}),-0.10(\mathrm{~s}, 9 \mathrm{H}) ;{ }^{13} \mathrm{C}$ NMR $\left(101 \mathrm{MHz} \mathrm{CDCl}_{3}\right) \delta 157.8,155.0,149.0,141.2,136.2,133.1,129.6,127.9,127.3,122.1,122.0$, -0.04; Anal. Calcd for $\mathrm{C}_{16} \mathrm{H}_{19} \mathrm{NSi}$ : C, 75.83; H, 7.56. Found: C, 75.59; H, 7.57.

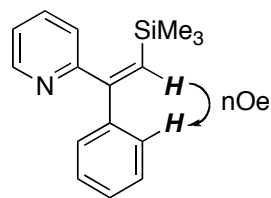

(Z)-3ae

(Z)-2-[1-Phenyl-2-(trimethylsilyl)ethenyl]pyridine [(Z)-3ae]. A colorless oil, $\mathrm{R}_{\mathrm{f}}$ 0.40 (hexane-ethyl acetate $=9: 1) .{ }^{1} \mathrm{H} \mathrm{NMR}\left(400 \mathrm{MHz}, \mathrm{CDCl}_{3}\right) \delta 8.65(\mathrm{ddd}, J=4.9$, $1.8,1.0 \mathrm{~Hz}, 1 \mathrm{H}), 7.65(\mathrm{td}, J=10.9,1.9 \mathrm{~Hz}, 1 \mathrm{H}), 7.33-7.18(\mathrm{~m}, 7 \mathrm{H}), 6.34(\mathrm{~s}, 1 \mathrm{H})$, $-0.03(\mathrm{~s}, 9 \mathrm{H}) ;{ }^{13} \mathrm{C} \mathrm{NMR}\left(101 \mathrm{MHz}, \mathrm{CDCl}_{3}\right) \delta 159.8,155.5,148.8,142.3,135.7$, 133.5, 128.0, 127.5, 127.4, 124.2, 122.2, 0.3; Anal. Calcd for $\mathrm{C}_{16} \mathrm{H}_{19} \mathrm{NSi}$ : C, 75.83; H, 7.56. Found: C, 76.04; H, 7.71. 
Determination of the regiochemistry of 3ae by protodesilylation. A solution of TBAF in THF $(1.0 \mathrm{M}, 0.24 \mathrm{~mL}, 0.24 \mathrm{mmol})$ was added to $(E)-3 a e(31 \mathrm{mg}, 0.12 \mathrm{mmol})$ dissolved in THF $(2.5 \mathrm{~mL})$. The resulting solution was heated at $60{ }^{\circ} \mathrm{C}$ for $8 \mathrm{~h}$ and extracted with ethyl acetate. The combined organic layers were washed with brine, dried over anhydrous $\mathrm{Na}_{2} \mathrm{SO}_{4}$, and then concentrated in vacuo. The residue was purified by flush chromatography on silica gel to give 2-(1-phenylvinyl)pyridine in $81 \%$ yield as a colorless oil. The ${ }^{1} \mathrm{H}$ NMR and ${ }^{13} \mathrm{C}$ NMR spectra of the obtained product was identical

with those reported in a literature. ${ }^{4}$ The same procedure using (Z)-3ae also gave the identical product quantitatively.

\section{References}

1. Pangborn, A. B.; Giardello, M. A.; Grubbs, R. H.; Rosen, R. K.; Timmers, F. J. Organometallics 1996, 15, 1518.

2. Cho, J.-W.; Lim, S.-C.; Maeng, C.-Y.; Hwang, S.-G.; Bae, S.-J.; Kim, E.-A. WO. Patent 2006075888.

3. Kanyiva, K. S.; Nakao, Y.; Hiyama, T. Angew. Chem., Int. Ed. 2007, 46, 8872.

4. Renninson, D.; Bova, S.; Cavalli, M.; Ricchelli, F.; Zullian, A.; Hopkins, B.; Brimple, M. A. Bioorg. Med. Chem. 2007, 15, 2963. 


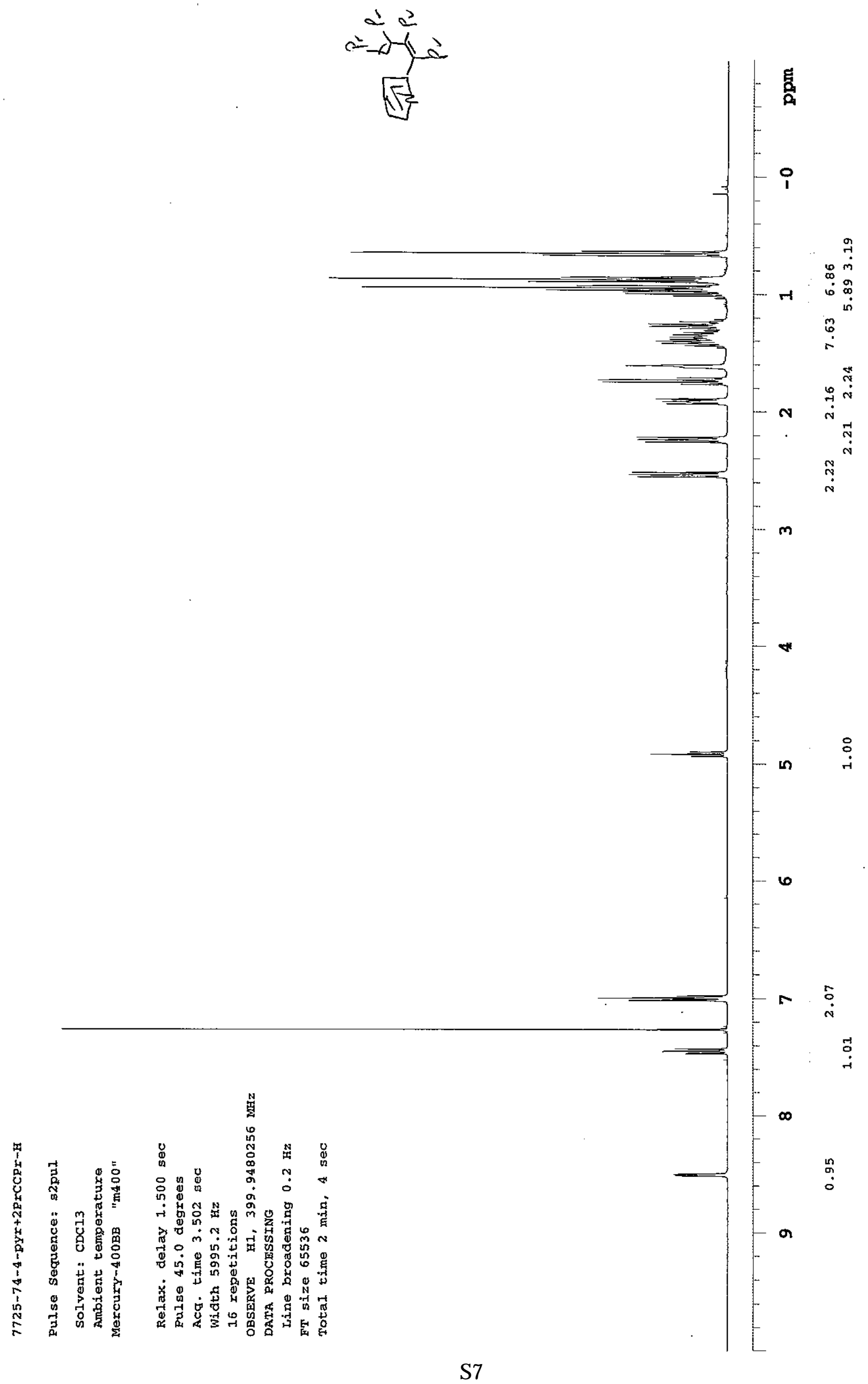



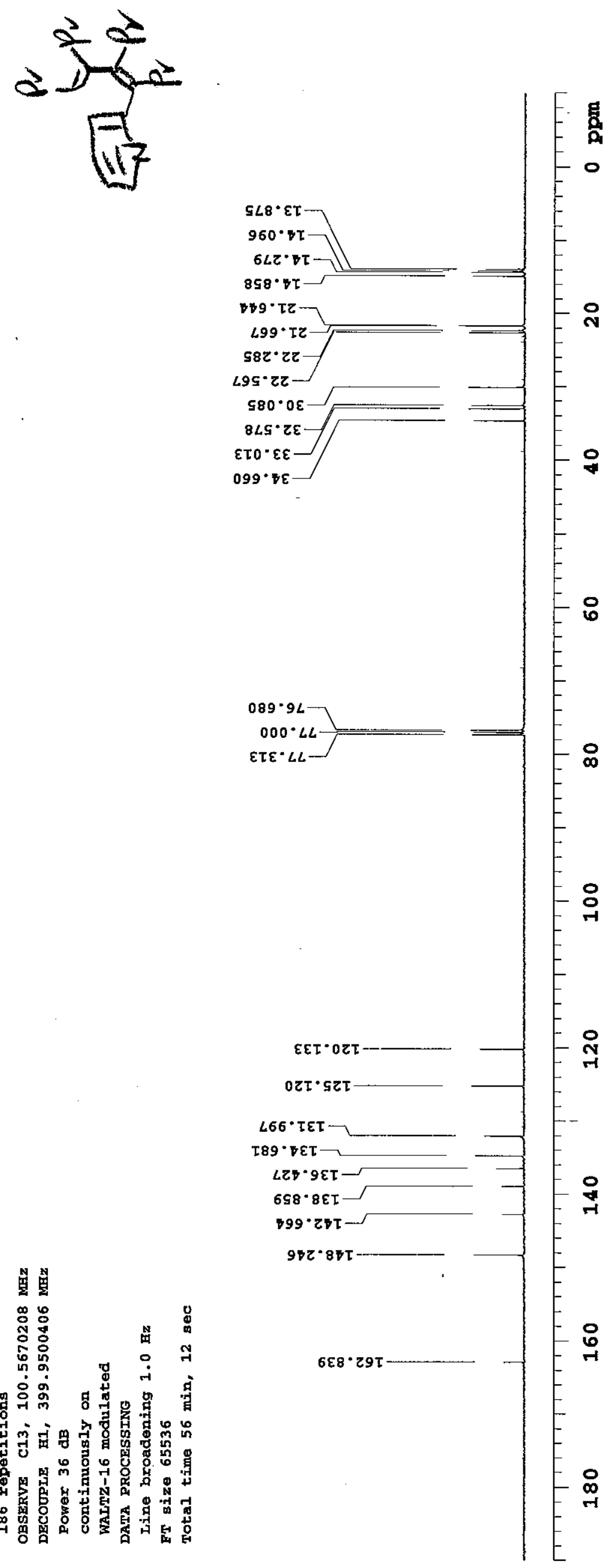


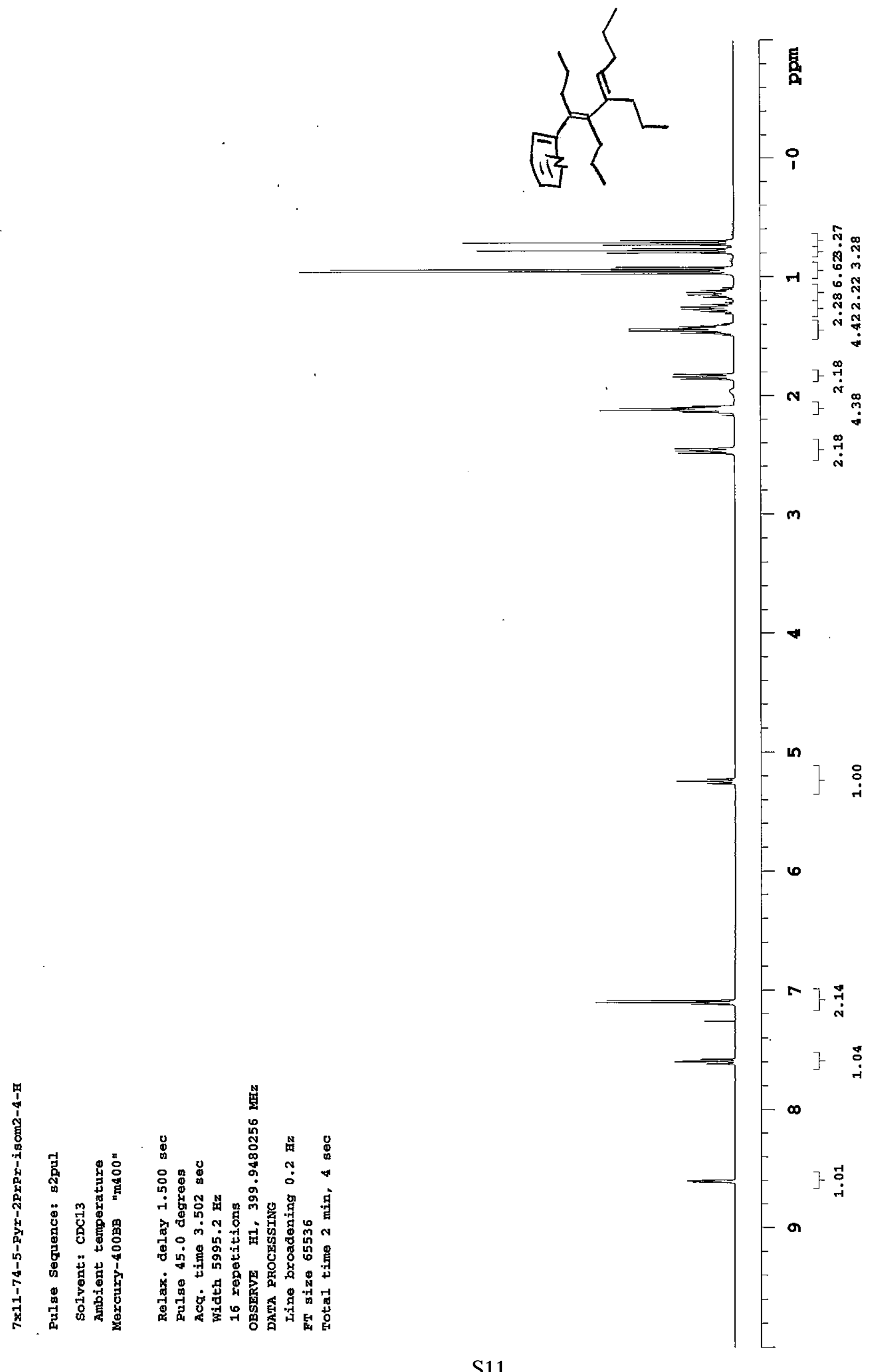




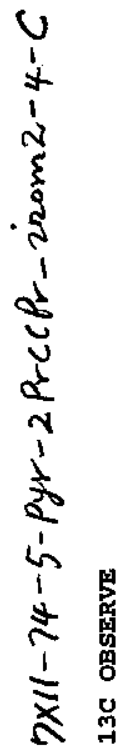
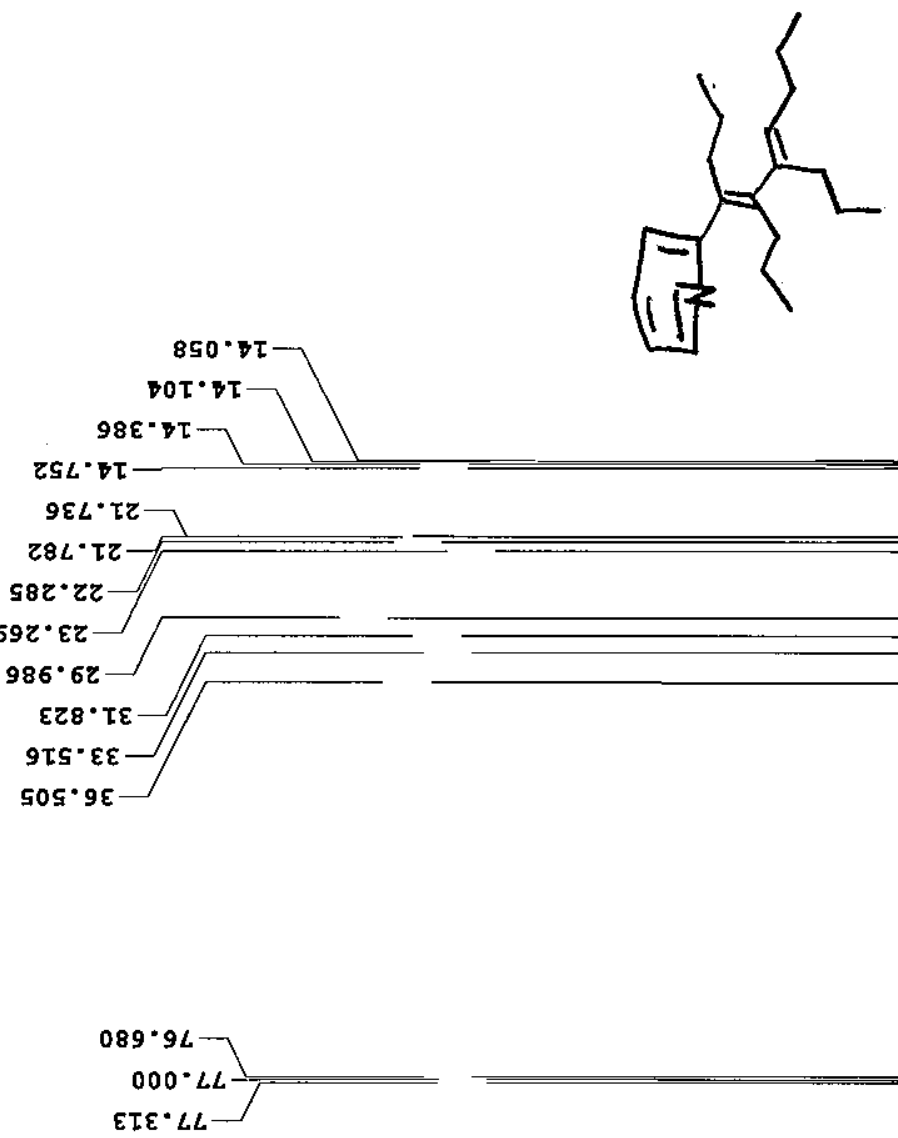

SEL'OZT

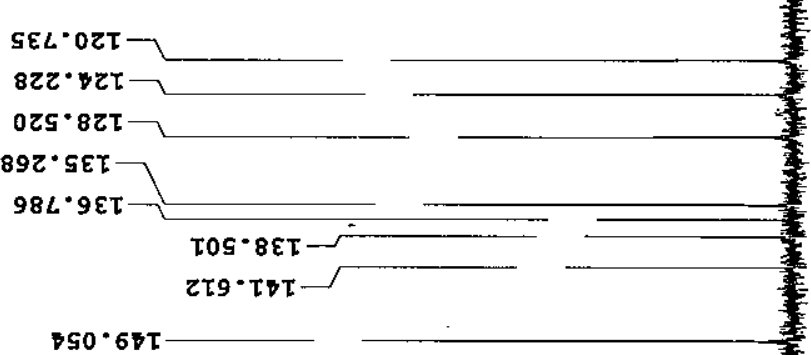

- 옥
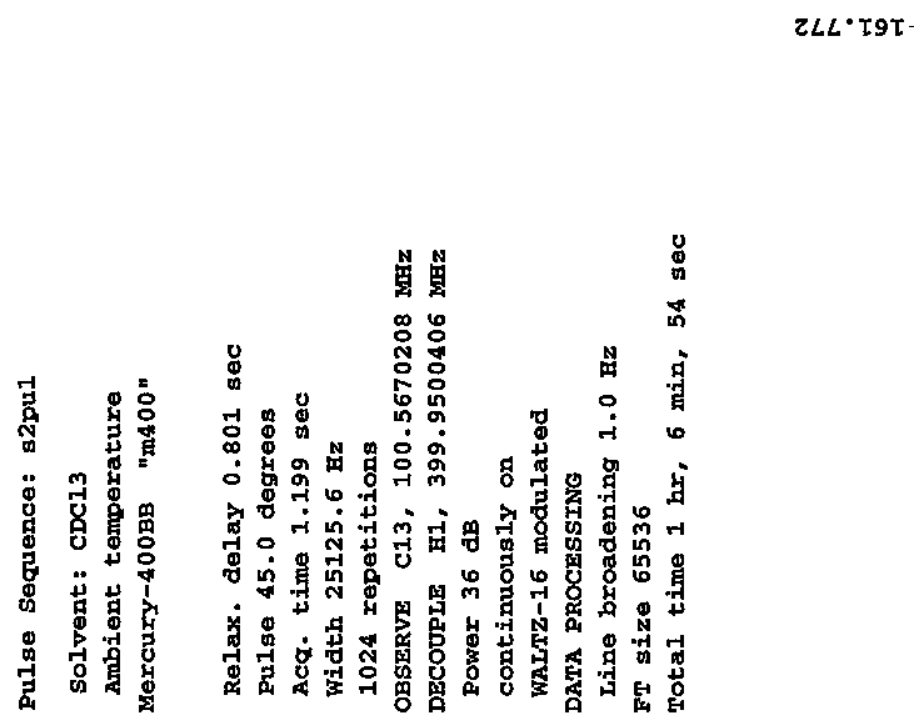

웅

$\stackrel{\infty}{\infty}$ 


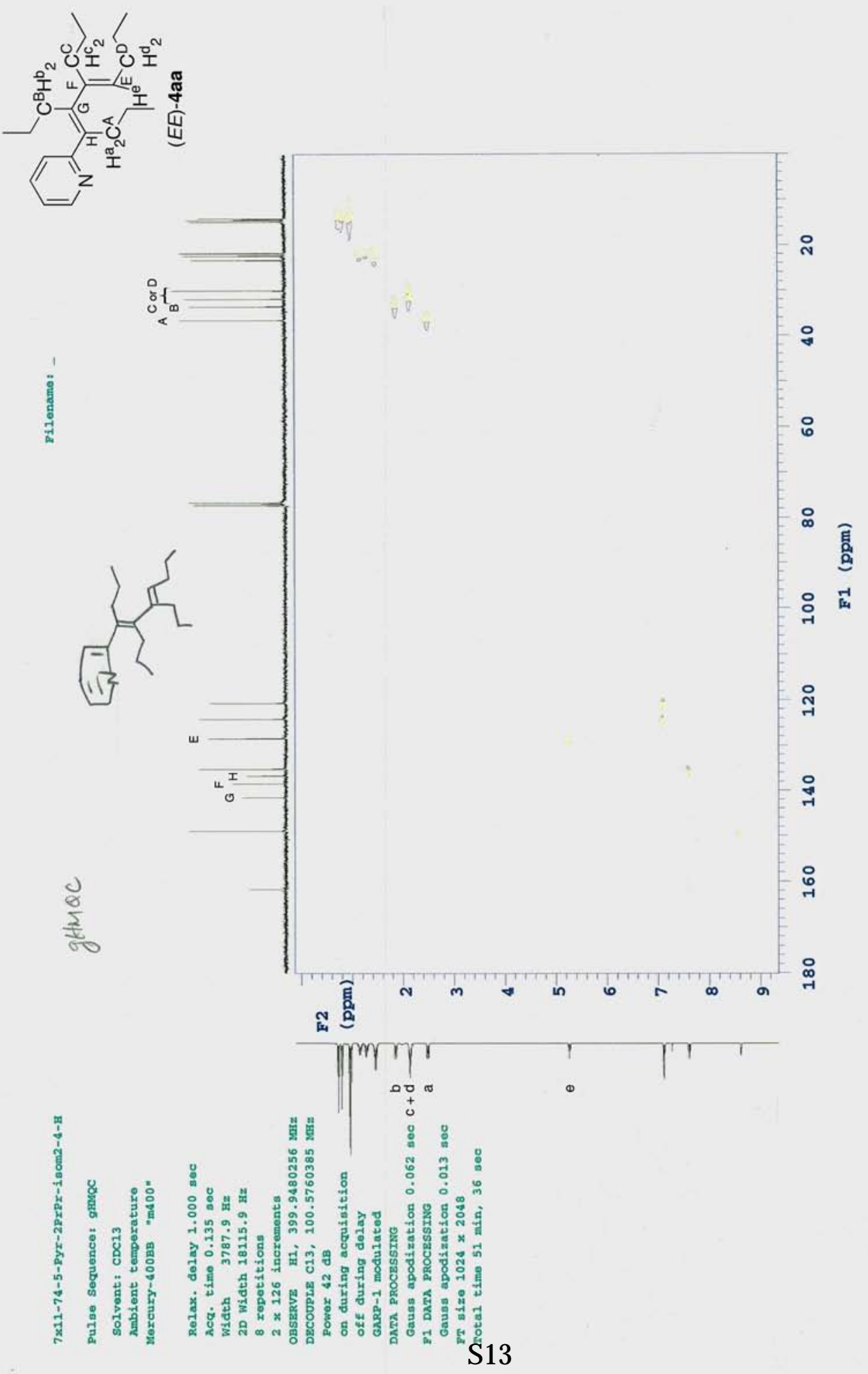




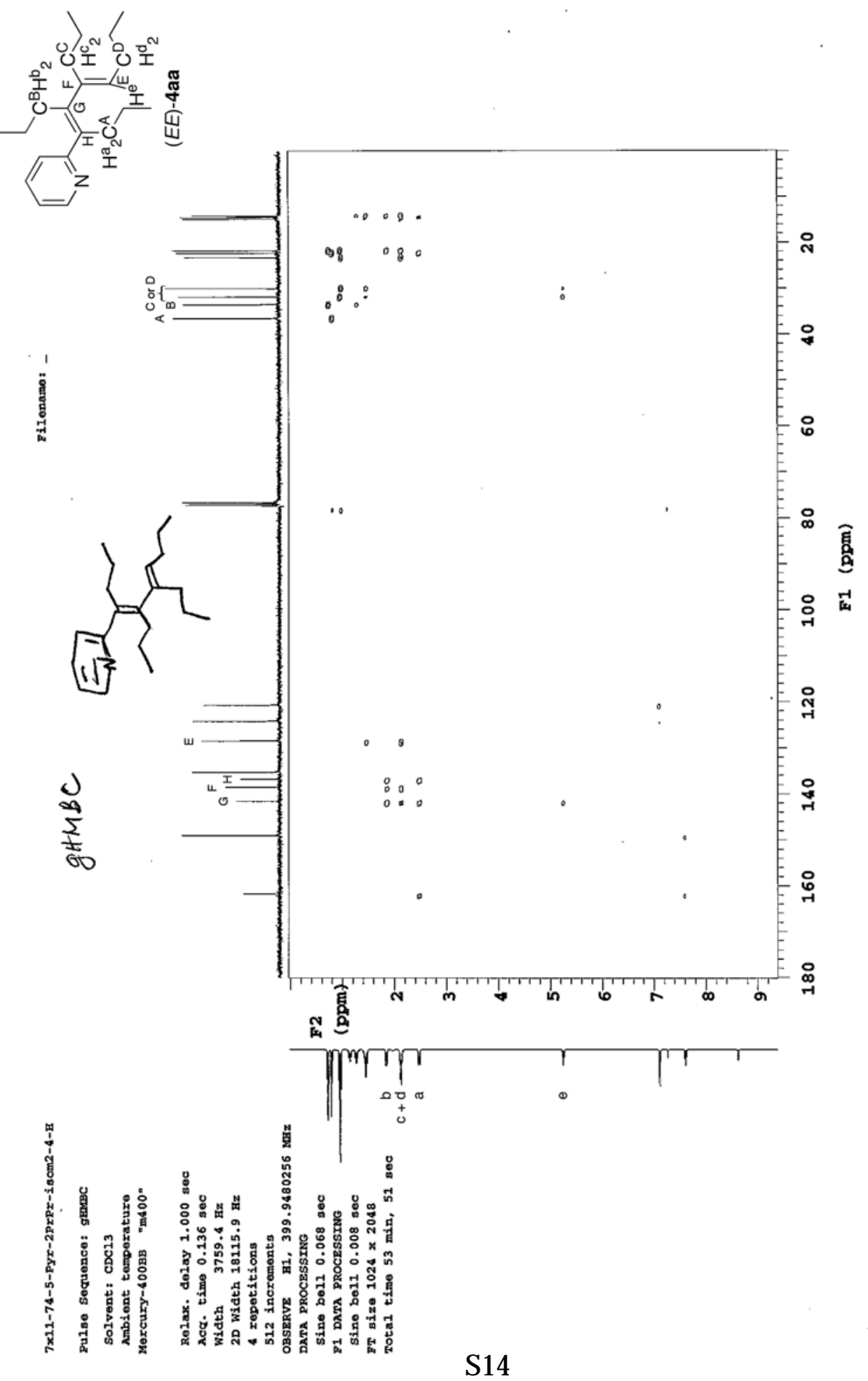



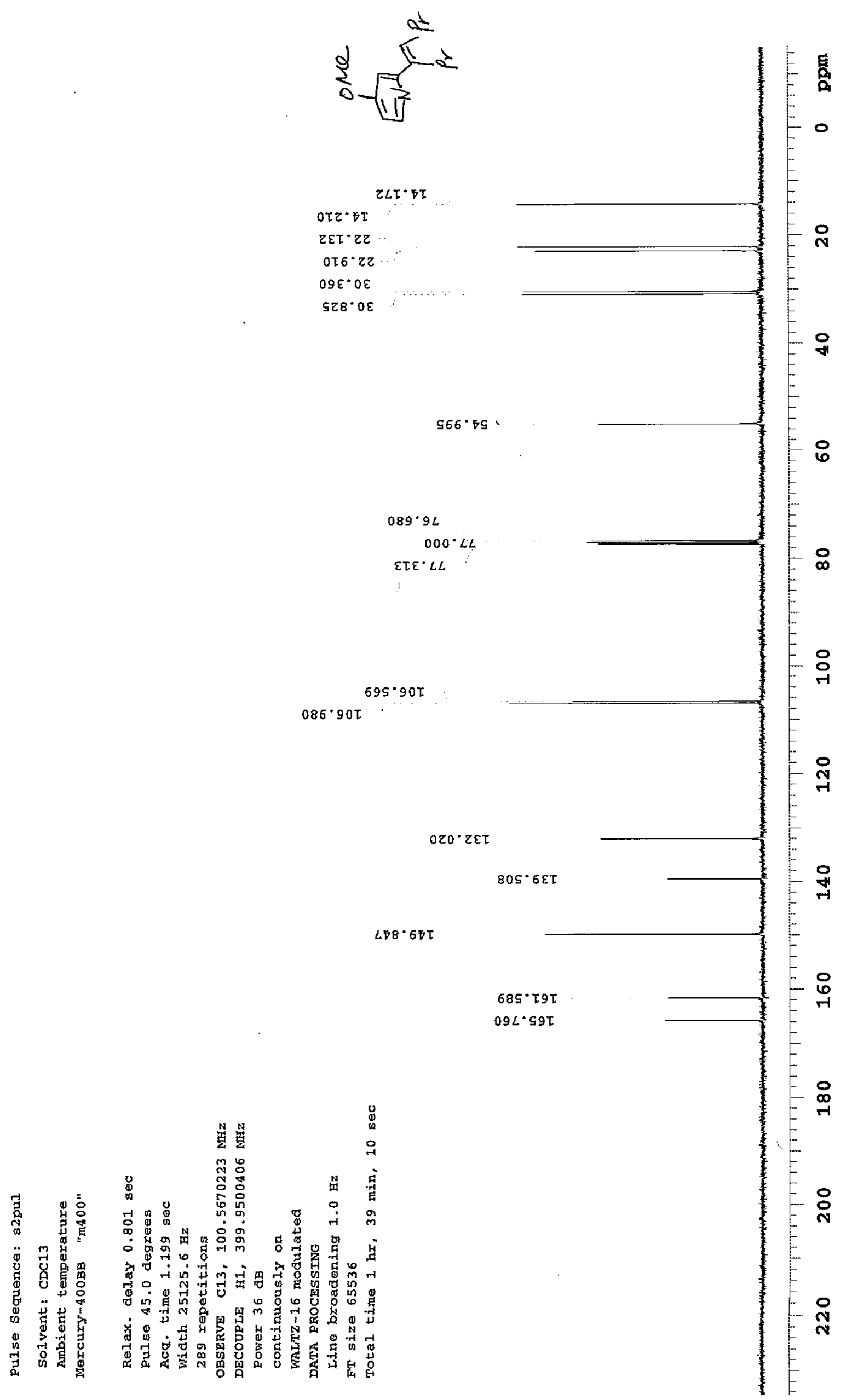

$\angle \nabla 8 \cdot 67 T$

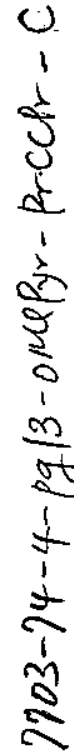

$685^{\circ}$ โ 9 โ

$09 L .59$ L

$0 z 0^{\circ} z \varepsilon \tau$

$805^{\circ} 6 \varepsilon T$

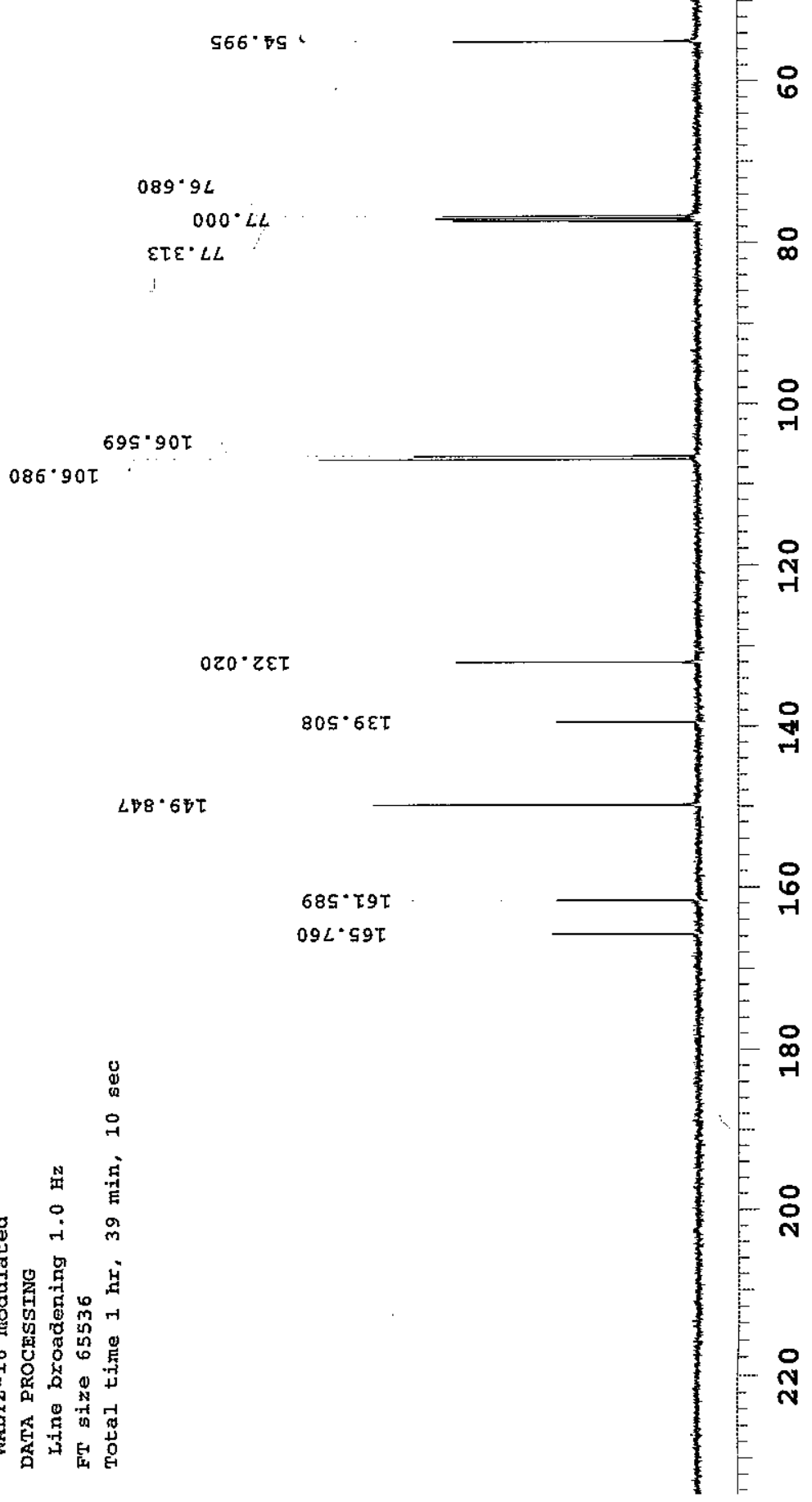




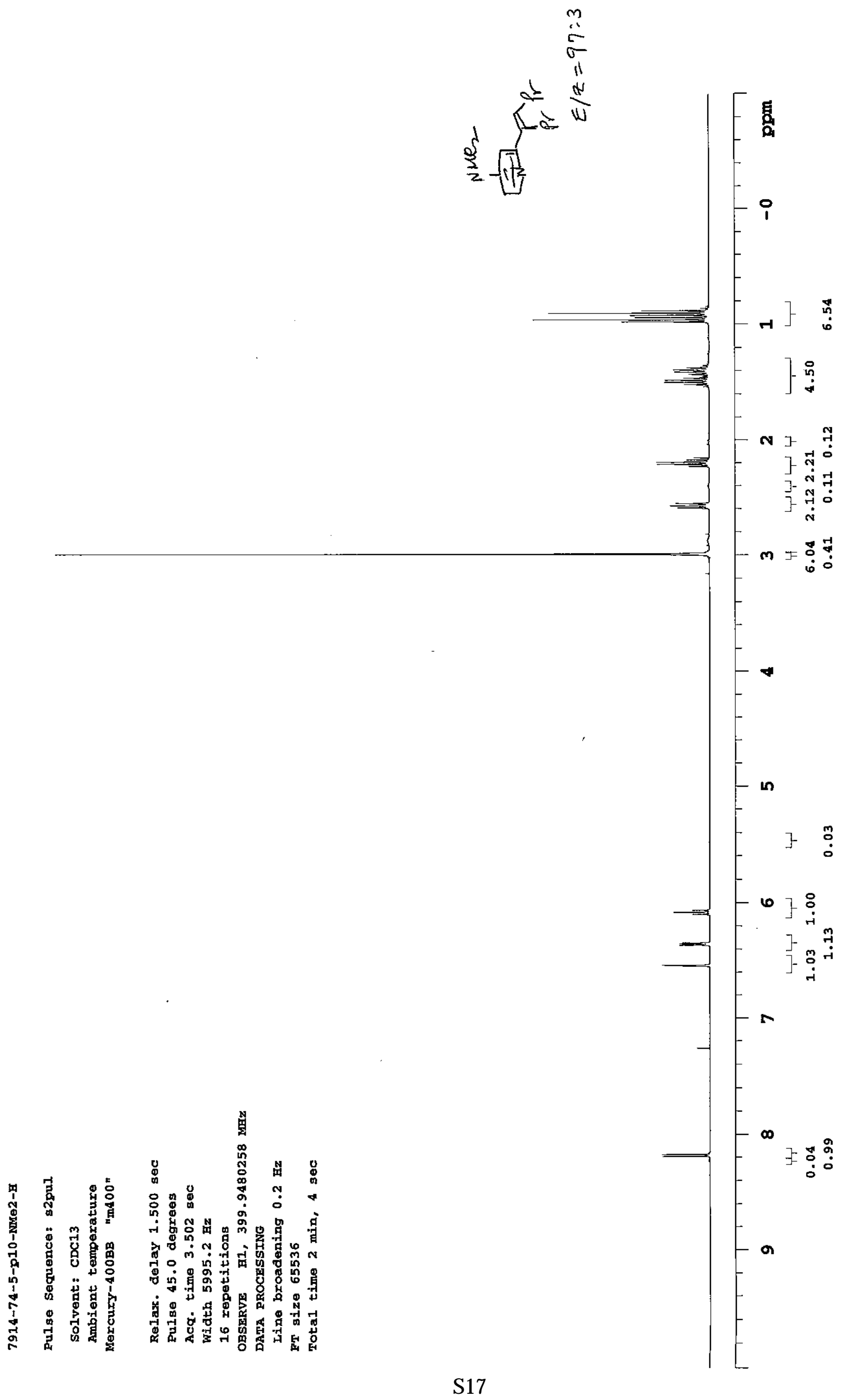




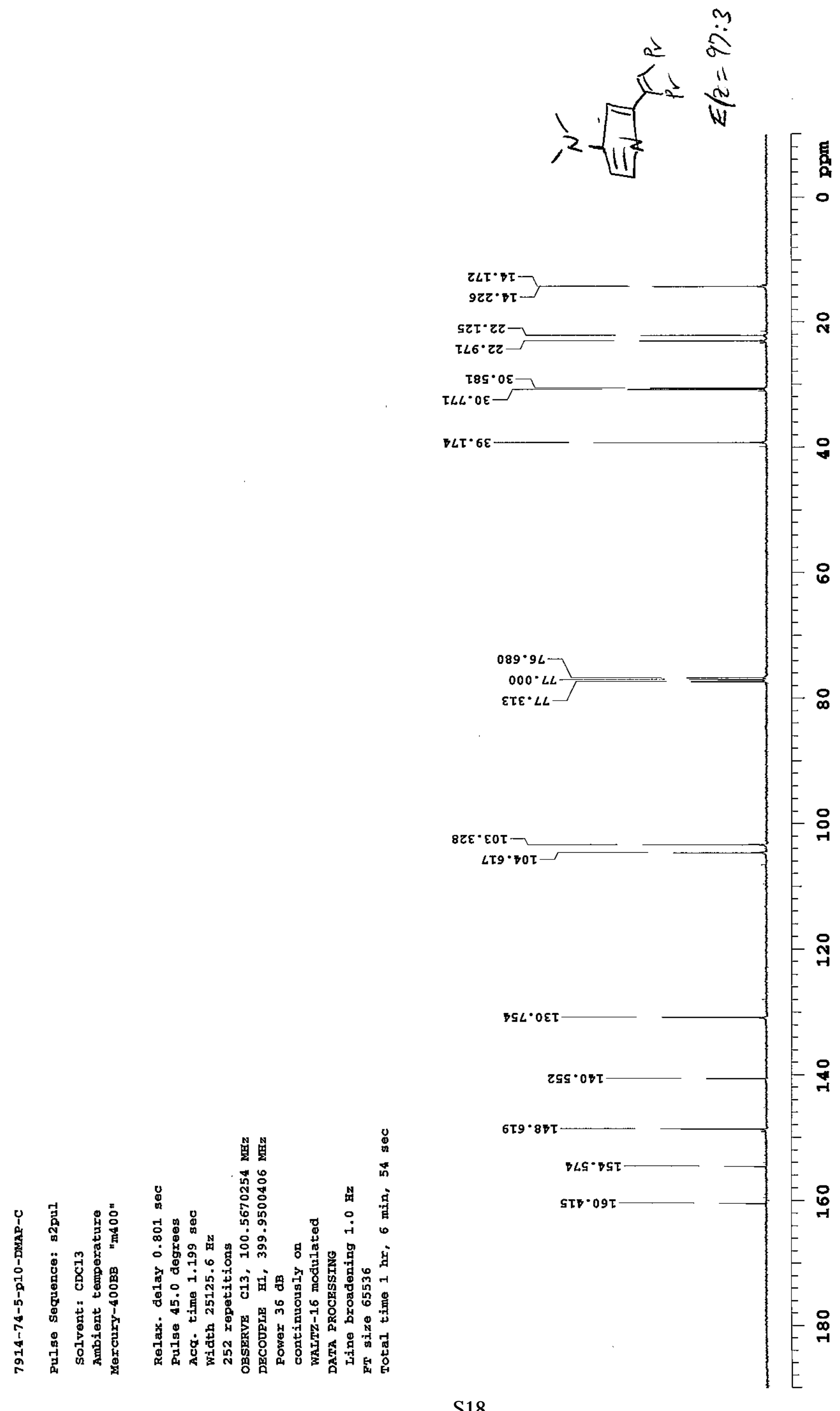




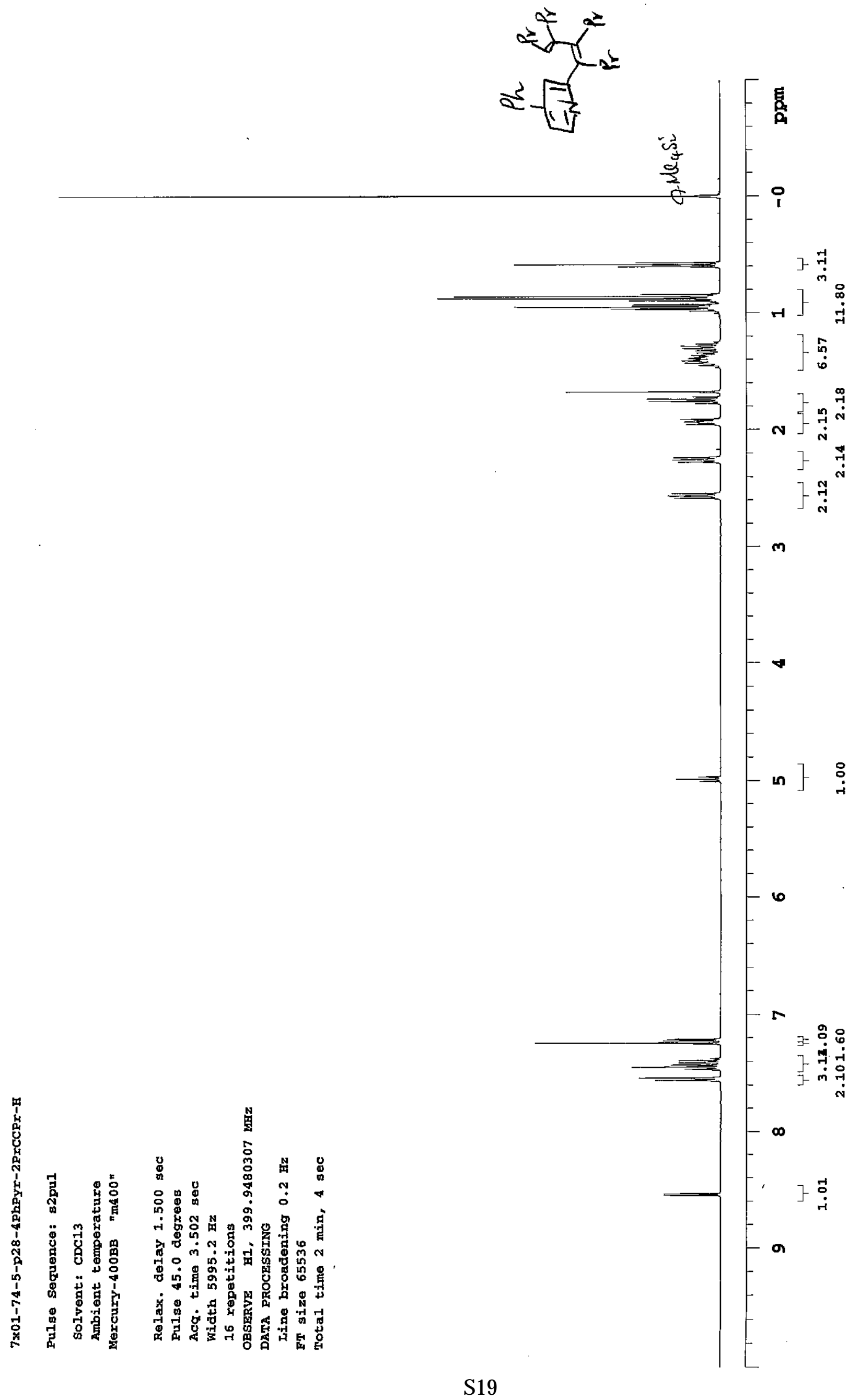




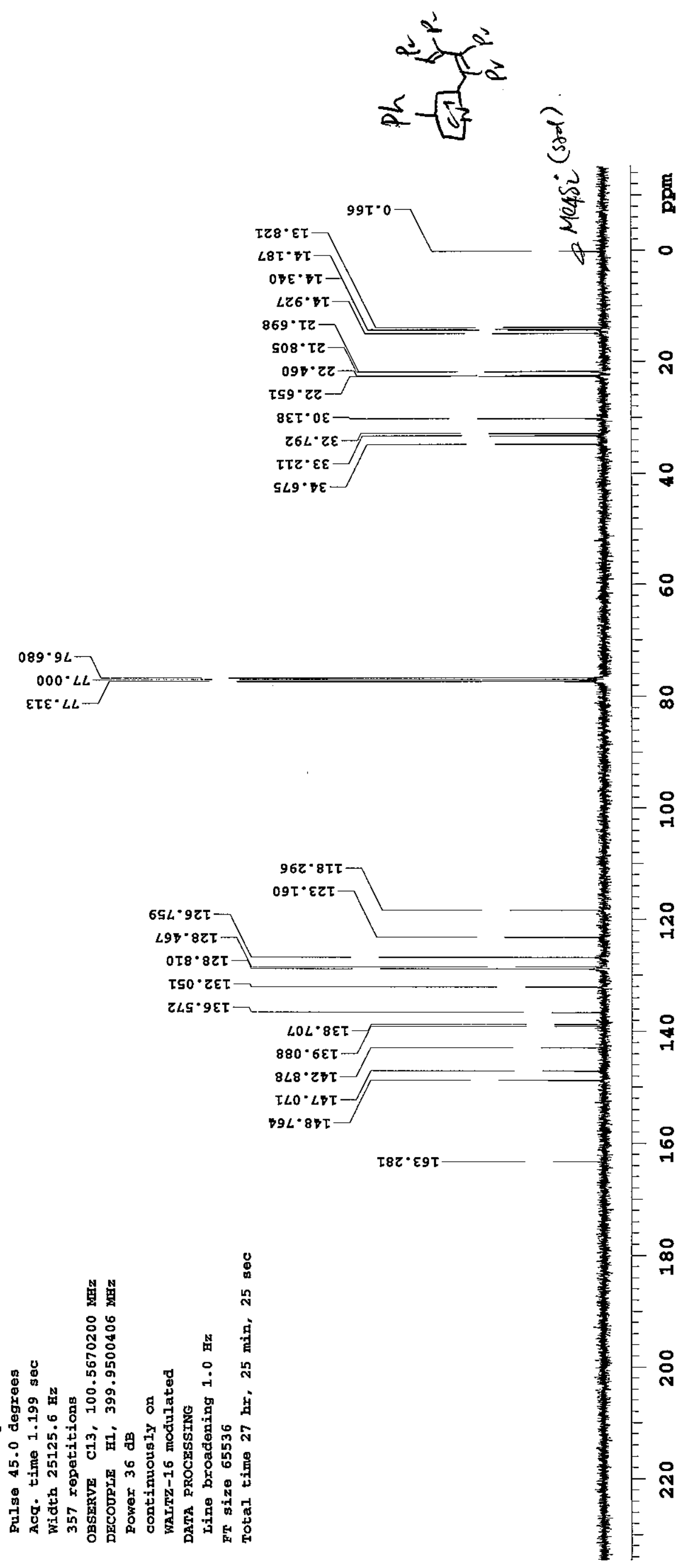




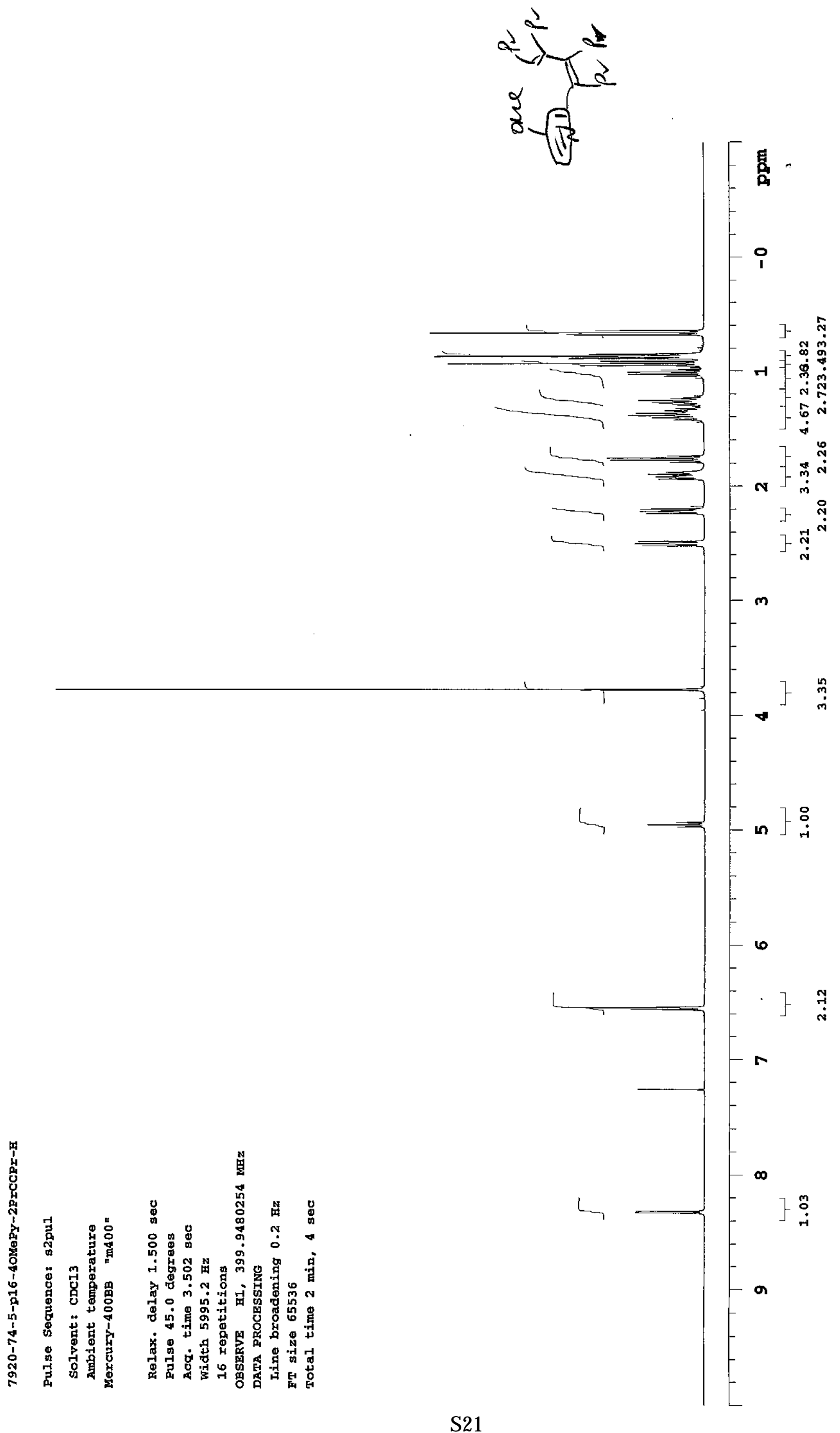




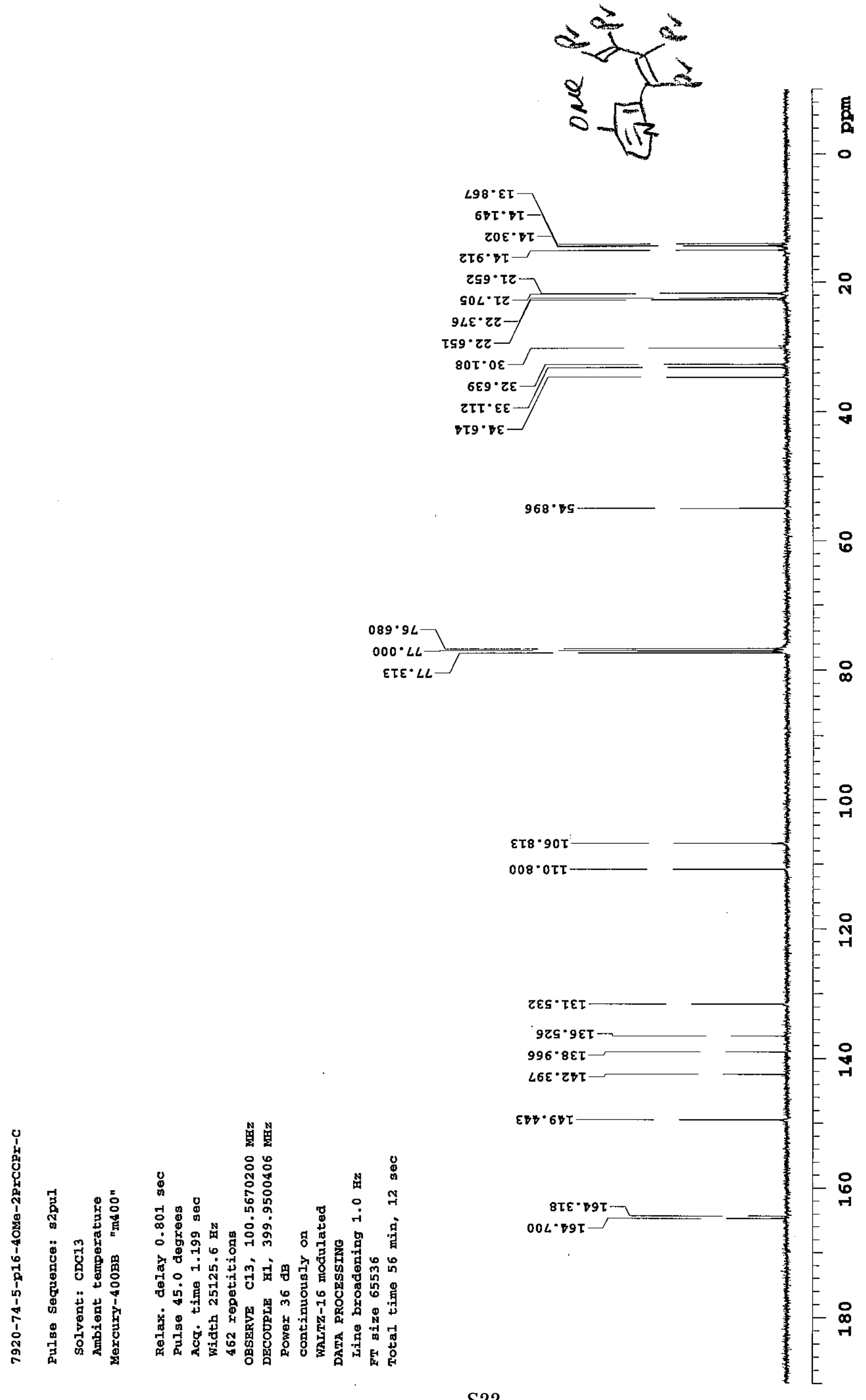



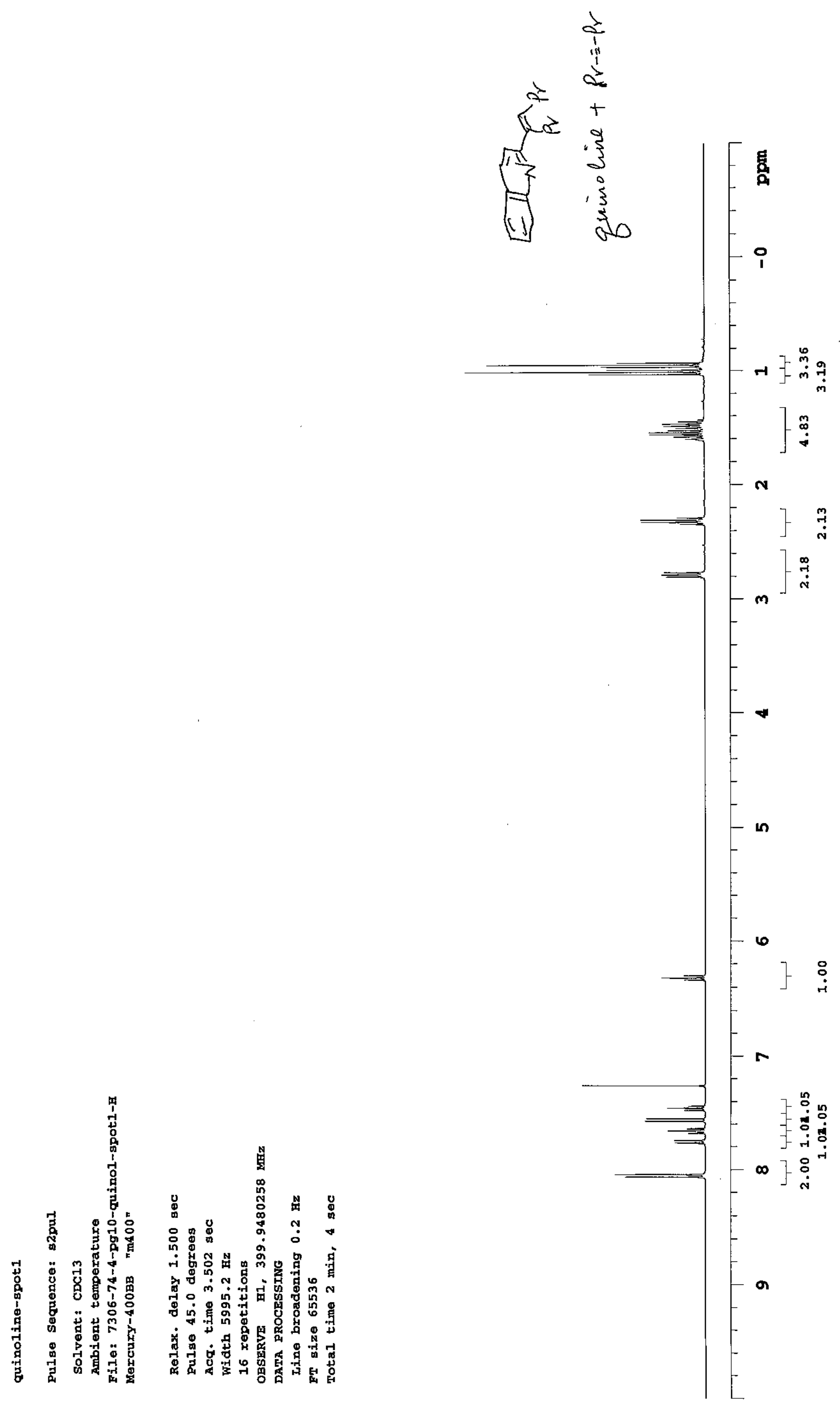


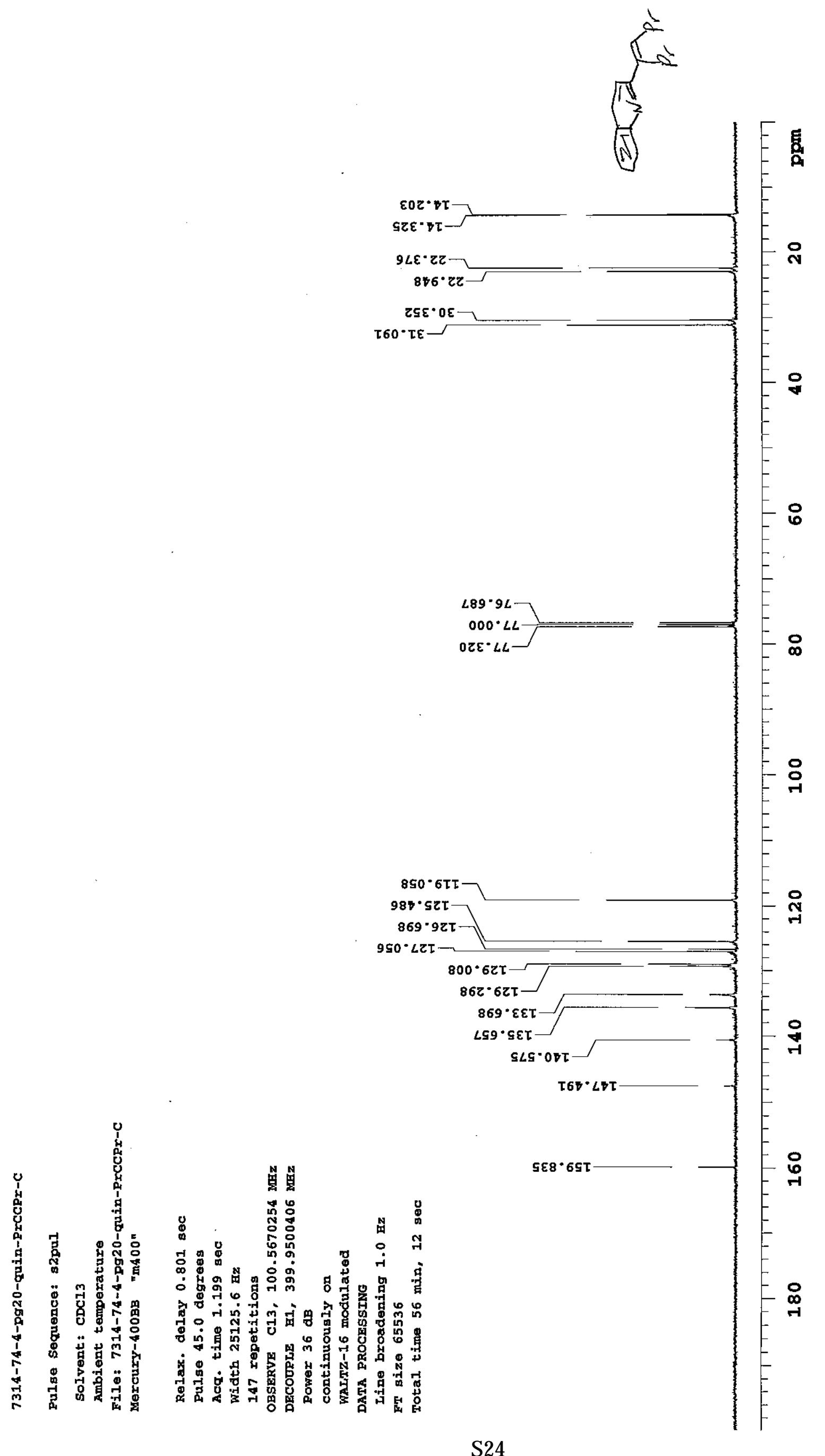




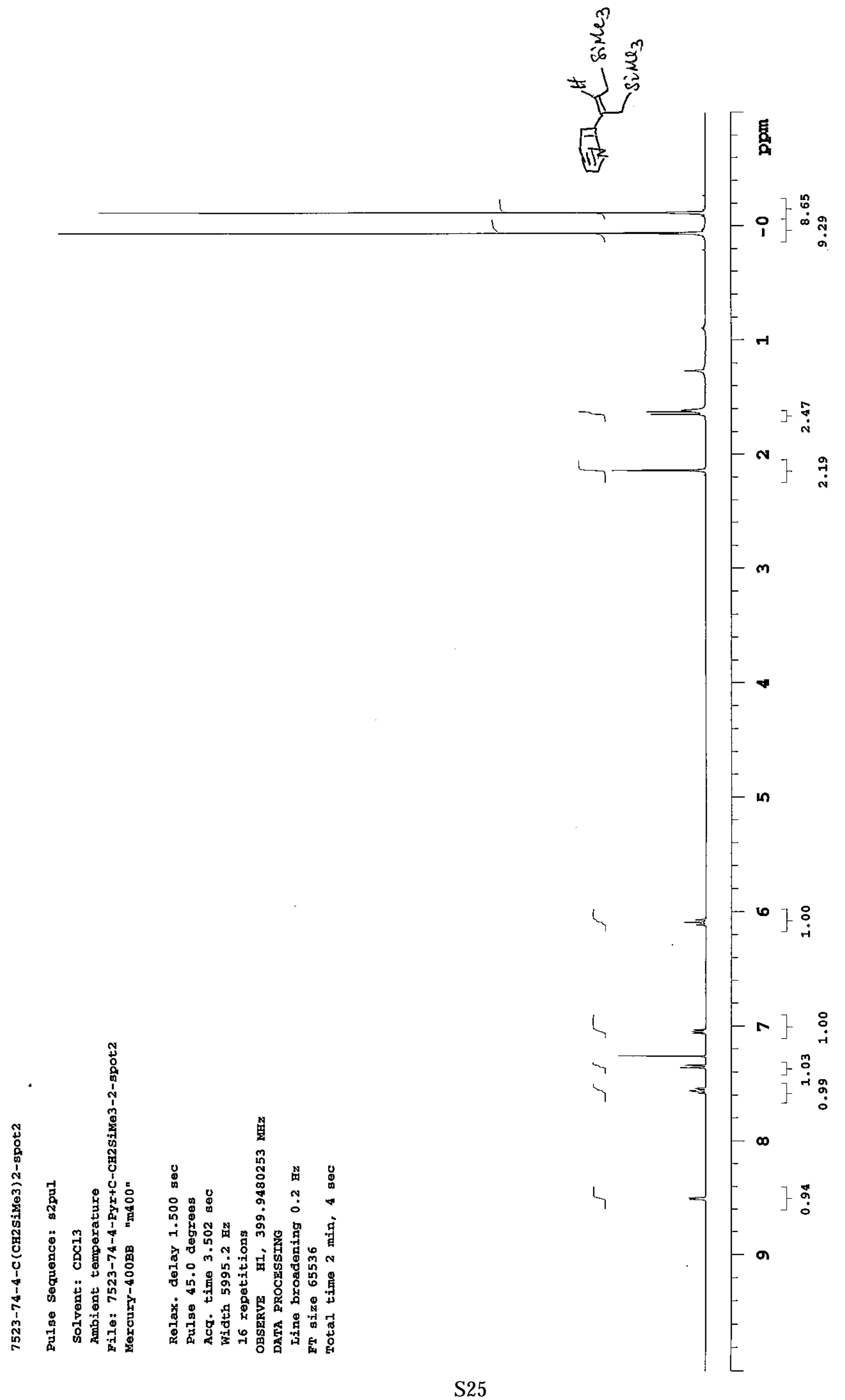




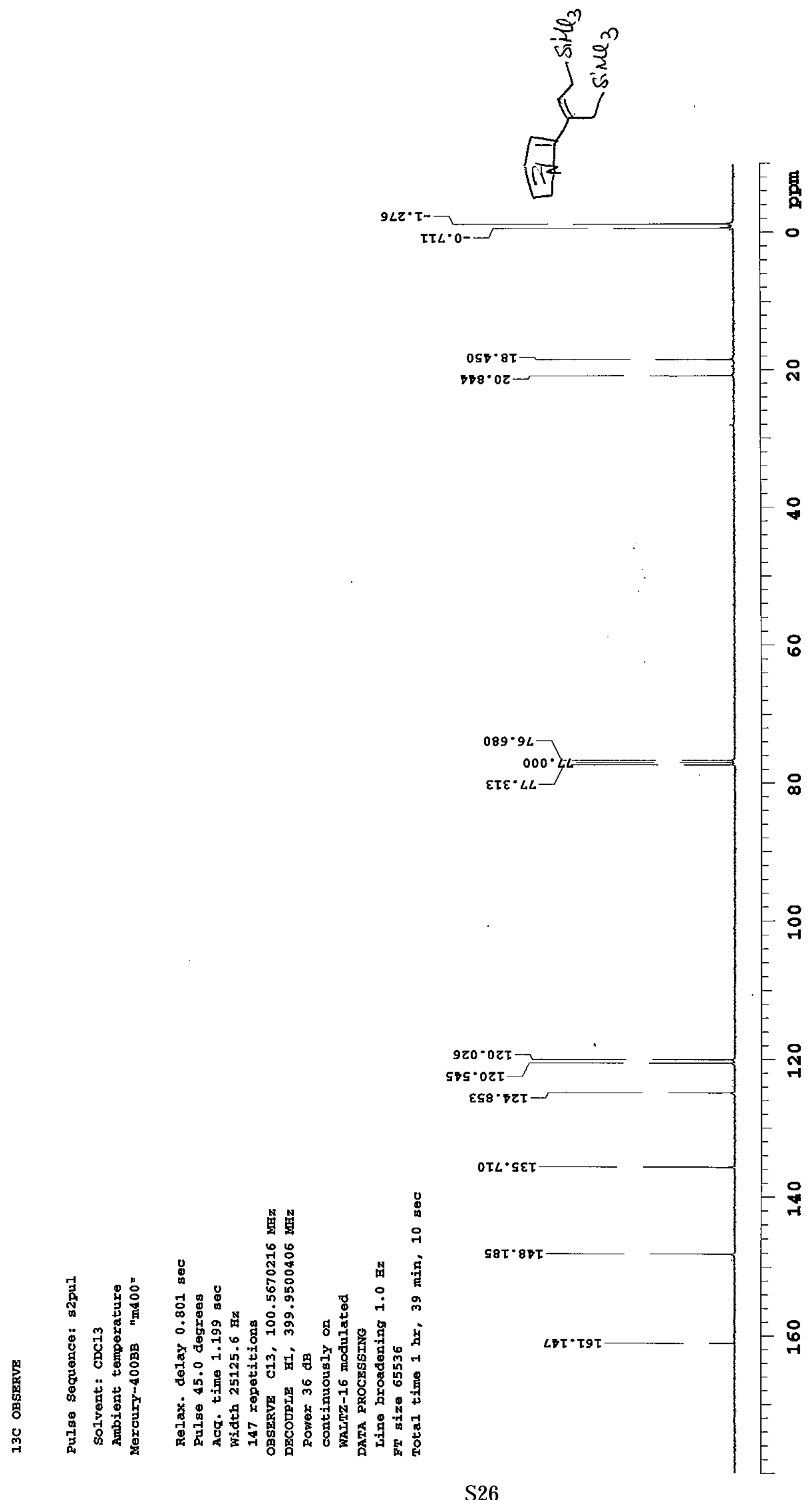




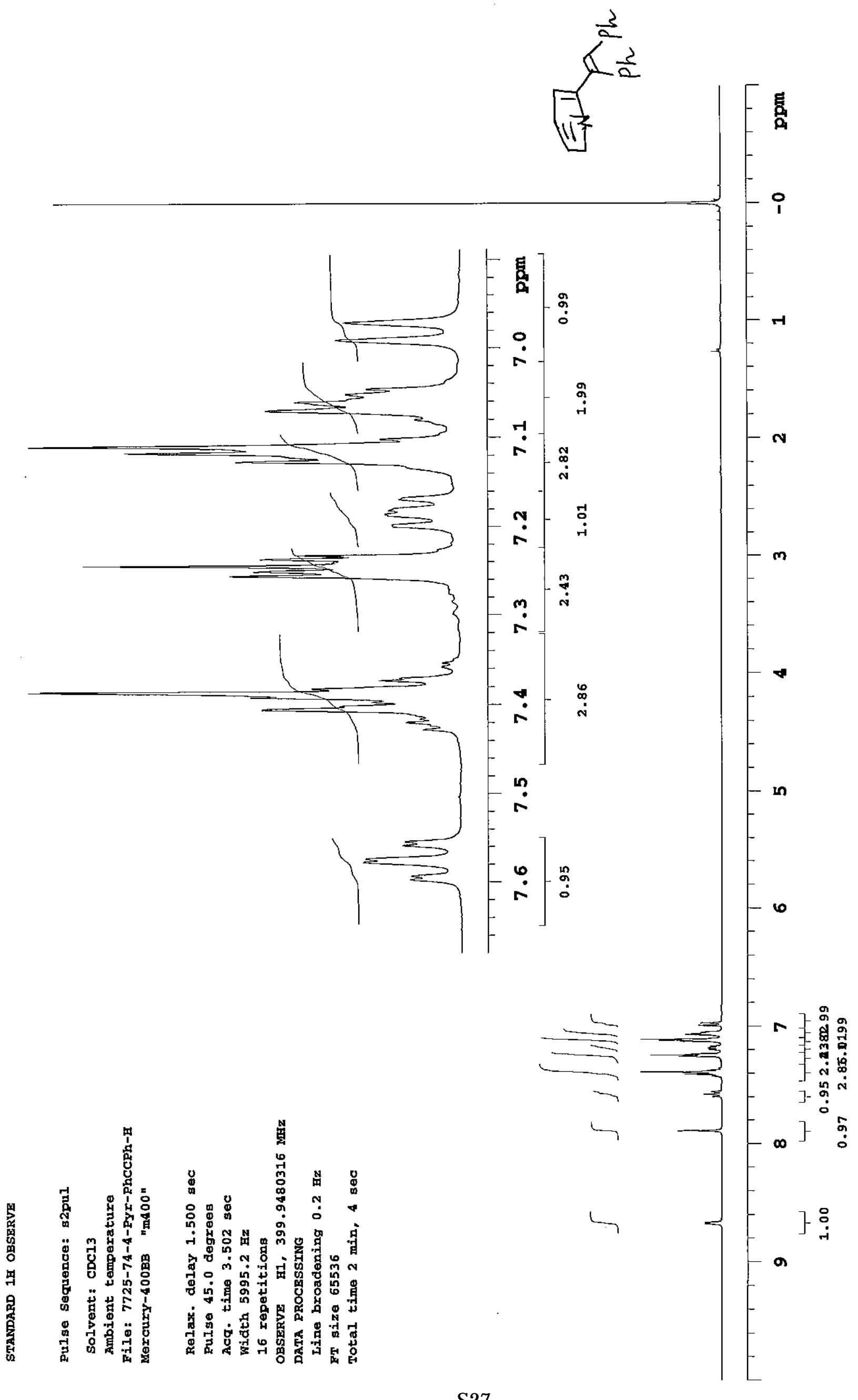




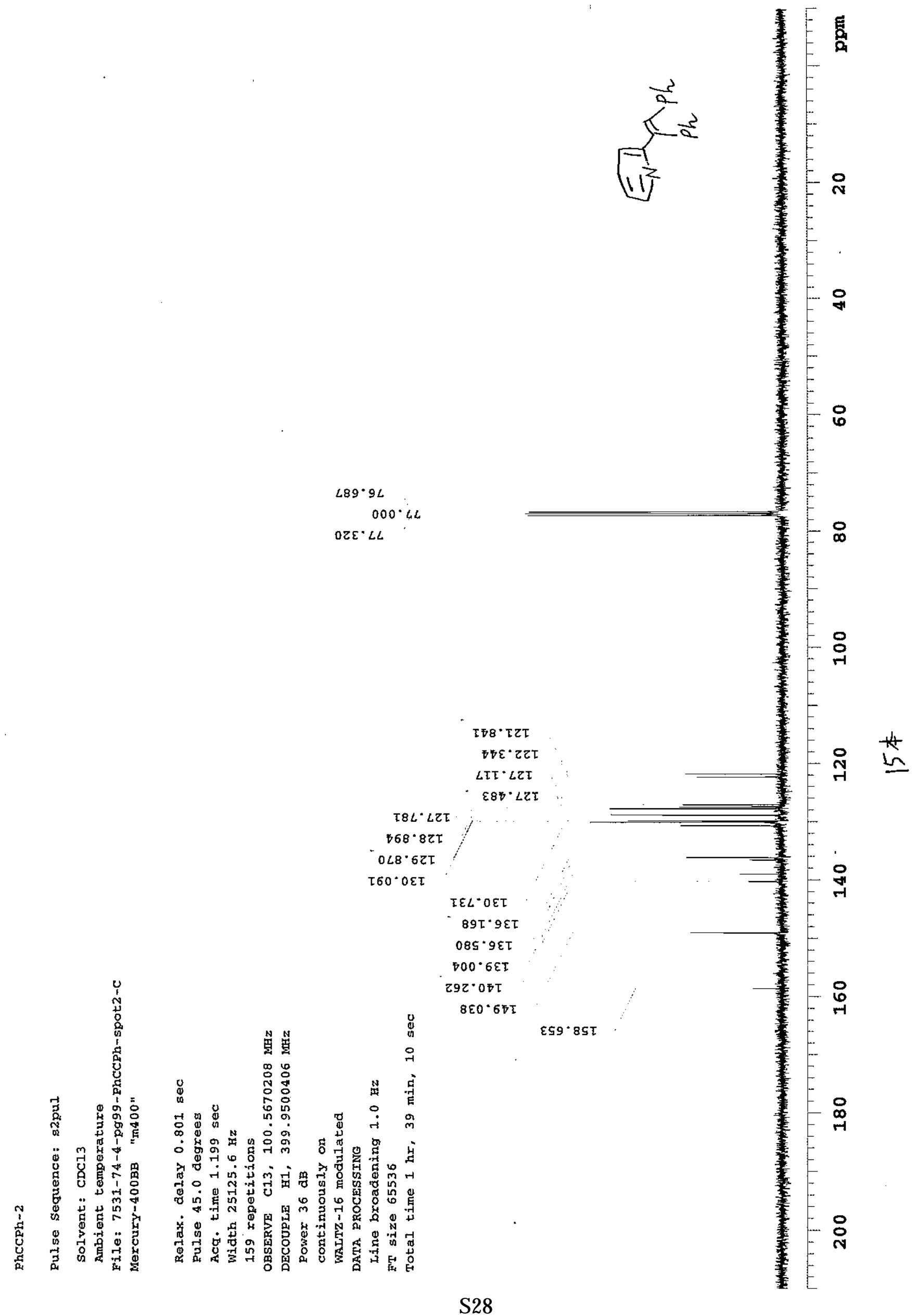

